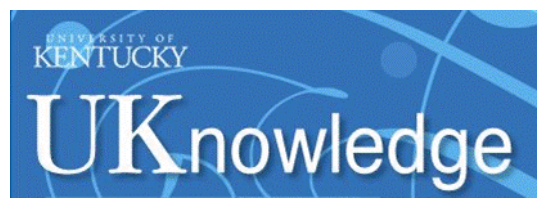

University of Kentucky

UKnowledge

\title{
The Chemical Enrichment of Gas in Broad Absorption Line QSOs: Rapid Star Formation in the Early History of Galaxies
}

\author{
Kirk Korista \\ University of Kentucky \\ Fred Hamann \\ University of California - San Diego \\ Jason W. Ferguson \\ University of Kentucky \\ Gary J. Ferland \\ University of Kentucky, gary@uky.edu
}

Follow this and additional works at: https://uknowledge.uky.edu/physastron_facpub

Part of the Astrophysics and Astronomy Commons, and the Physics Commons

Right click to open a feedback form in a new tab to let us know how this document benefits you.

\section{Repository Citation}

Korista, Kirk; Hamann, Fred; Ferguson, Jason W.; and Ferland, Gary J., "The Chemical Enrichment of Gas in Broad Absorption Line QSOs: Rapid Star Formation in the Early History of Galaxies" (1996). Physics and Astronomy Faculty Publications. 151.

https://uknowledge.uky.edu/physastron_facpub/151

This Article is brought to you for free and open access by the Physics and Astronomy at UKnowledge. It has been accepted for inclusion in Physics and Astronomy Faculty Publications by an authorized administrator of UKnowledge. For more information, please contact UKnowledge@lsv.uky.edu. 


\section{The Chemical Enrichment of Gas in Broad Absorption Line QSOs: Rapid Star Formation in the Early History of Galaxies}

\section{Digital Object Identifier (DOI)}

http://dx.doi.org/10.1086/177091

\section{Notes/Citation Information}

Published in The Astrophysical Journal, v. 461, no. 2, p. 641-656.

(c) 1996. The American Astronomical Society. All rights reserved.

The copyright holder has granted permission for posting the article here. 


\title{
THE CHEMICAL ENRICHMENT OF GAS IN BROAD ABSORPTION LINE QSOs: RAPID STAR FORMATION IN THE EARLY HISTORY OF GALAXIES
}

\author{
Kirk Korista, ${ }^{1,2}$ Fred Hamann, ${ }^{3}$ Jason Ferguson, ${ }^{1}$ and Gary Ferland ${ }^{1}$ \\ Received 1995 January 9 ; accepted 1995 October 24
}

\begin{abstract}
Utilizing spectra from ground-based and Hubble Space Telescope observations, Korista et al. identified at least 12 broad absorption lines in the broad absorption line (BAL) QSO $0226-1024\left(z_{\mathrm{em}}=2.256\right)$, many for the first time. In the present paper we use seven of their measured column densities to investigate the BAL gas ionization equilibrium and metallicity. We assume that the metal abundances follow the rapid star formation (RSF) models of Hamann \& Ferland or are simply scaled from solar ratios to (1) limit the free parameters in constraining the overall metallicity and (2) test the applicability of such a scenarios to the enrichment of BAL gas. Photoionization calculations which consider separately the highly ionized gas ( $\mathrm{C}$ IV, $\mathrm{N}$ v, and $\mathrm{O}$ vI) and the doubly ionized metal gas ( $\mathrm{C}$ III, $\mathrm{N}$ III, and $\mathrm{O}$ III), as well as those which consider the full set of ionic column densities, all indicate significant enhancements in the metal abundances over solar, up to $\sim 10 \mathrm{Z}_{\odot}$. The requirement for greater than solar metallicities is shown to be independent of the large range of ionizing continuum shapes and of the adopted enrichment scenario, and even larger metallicities could be derived if the metals are allowed to vary independently in a non-RSF enrichment scenario. Because the BALs in 0226-1024 that are measurable from the ground have column densities typical in BAL QSOs, the main result, $Z>Z_{\odot}$, should apply generally to the BAL QSO phenomenon. This result supports an enrichment scenario expected in the cores of massive galaxies in which early rapid star formation produces metal enriched gas.

Metallicities enhanced over solar will increase the opacity of the outflowing BAL gas without significantly increasing the mass load of whatever is driving the outflow. This should have a significant impact on radiative acceleration models of BAL outflows which have, up to now, assumed solar metallicity.

Subject headings: galaxies: abundances - galaxies: evolution — galaxies: stellar content quasars: absorption lines - quasars: general
\end{abstract}

\section{INTRODUCTION}

High-velocity outflowing gas is observed in $\sim 10 \%$ of optically selected quasars, such as in the Large Bright Quasar Survey (LBQS) (Weymann et al. 1991, hereafter W91; Foltz et al. 1987, 1989; Chaffee et al. 1991; Morris et al. 1991). The signature of this gas is the presence of blueshifted, broad $\left(\sim 10,000 \mathrm{~km} \mathrm{~s}^{-1}\right)$ resonance line absorption, and quasars exhibiting these features are known as broad absorption line quasi-stellar objects (BAL QSOs) (see Korista et al. 1993 for an atlas of 72 C IV BAL QSOs). Because of their extreme velocity widths and high stages of ionization, it is believed that the outflows are intimately connected to the central engines of QSOs. Furthermore, it appears that all BAL QSOs are radio quiet at a statistically significant level (Stocke et al. 1992). There is also mounting evidence that the high-velocity outflows are present in most or all radio-quiet QSOs, and that the source covering fraction of the absorbing gas dictates the fraction of all radioquiet QSOs that are observed as BAL QSOs (W91; Hamann, Korista, \& Morris 1993). The mass flow rate has been estimated to be several tenths to a few solar masses per year (see, for example, Turnshek 1988).

The origin of the BAL gas, the nature of its outflow, its ionization equilibrium, and chemical composition are outstanding questions. At least two (ionization and chemical composition) and possibly all of these questions are inter-

\footnotetext{
${ }^{1}$ Department of Physics and Astronomy, University of Kentucky, Lexington, KY 40506.

${ }^{2}$ korista@pa.uky.ed.

${ }^{3}$ Center for Astrophysics and Space Sciences, University of CaliforniaSan Diego, La Jolla, CA 92093.
}

connected. Until very recently, what we knew about the absorbing gas resulted from the study of mainly four BALs: H I $\lambda 1216(\mathrm{Ly} \alpha), \mathrm{C}$ IV $\lambda 1549, \mathrm{~N}$ v $\lambda 1240$, and Si IV $\lambda 1397$. In addition, roughly $10 \%$ of the observed BAL QSOs exhibit additional low-ionization BALs: Mg II $\lambda 2800, \mathrm{Al}$ II $\lambda 1671$, Al III $\lambda 1859$, and C II $\lambda 1335$ (see Voit, Weymann, \& Korista 1993). The large measured $N$ v column density relative to that of C IV, the possible identification of $P \mathrm{v} \lambda \lambda 1118,1128$ in at least two BAL QSOs $(1413+1143,0946+3009$, and possibly 0226 - 1024) (Turnshek et al. 1987; Junkkarinen et al. 1995; Korista et al. 1992), and the long-standing problem of the strength of the Si IV BAL (see Weymann, Turnshek, \& Christiansen 1985; hereafter WTC85) have led some investigators to speculate about the presence of supersolar metal abundances $\left(\mathrm{Z} \gtrsim 10 \mathrm{Z}_{\odot}\right)$ in BAL gas (Turnshek 1988; but see Kwan 1990). However, with generally so few BALs observable, our understanding of the chemical abundances and ionization of the outflowing gas has been sketchy, at best. This situation has begun to change dramatically with the Hubble Space Telescope (HST) observations of the BAL QSOs 0226-1024 $\left(z_{\mathrm{em}}=2.256\right)$ (Korista et al. 1992; hereafter K92) and $0946+3009\left(z_{\mathrm{em}}=1.223\right)$ (Junkkarinen et al. 1995) that have yielded column density measurements of C III, N III, N IV, O III, O IV, O vI, S IV, S v, S VI, and possibly Fe III and/or P V. As more of these types of observations are made, better constraints on the physical parameters which govern the BAL gas will come to light.

Most studies of the BAL region have come to the conclusion, still open to question, that it lies just outside of, or is a lower column density component of, the broad emissionline region (see Turnshek 1984). The origin and geometry of the gas in the vicinity of the central engine are uncertain, 
although recent progress has been made in the derivation of chemical abundances of QSO emission-line gas. Hamann \& Ferland (1992, 1993, hereafter HF92, HF93) and Ferland et al. (1996) showed that early epoch rapid star formation could supply gas with the enhanced metallicities they inferred from the observed emission-line ratios of $\mathrm{N} v$ to $\mathrm{C}$ IV and $\mathrm{He}$ II in high-redshift QSOs. High metallicities have been estimated for old stellar populations in the cores of nearby galaxies (Arimoto \& Yoshi 1986; Bica 1988; Bica, Arimoto, \& Alloin 1988) and in the center of our own Galaxy (Rich 1988; Geisler \& Friel 1992; McWilliam \& Rich 1994), indicating that rapid star formation does occur at early epochs in galactic evolution. Possible origins postulated for the BAL gas include the ablation of accretion disks and the stripping of material from evolved stellar atmospheres (see Murray et al. 1995; Roos 1992). Recent work by Scoville \& Norman (1995) postulates that BAL gas results from stellar "contrails" produced by circumstellar mass loss. In either case, the chemical abundances would reflect generations of stellar evolutions and are consistent with the HF93 model in which the lines are assumed to form in metal-rich "interstellar" gas.

In this paper we investigate the ionization equilibrium and chemical abundances of the BAL gas using the measured column density of BAL ions. Our goals are to estimate conservatively the metallicity of the BAL gas and to test the hypothesis that this gas results from the early-epoch rapid star formation (hereafter RSF) expected in galactic nuclei. The HF92 and HF93 calculations of explicit abundances as functions of metallicity provide a template for the RSF scenario. Ideally, we would derive the abundance of each element, $X / \mathrm{H}$ by simply comparing the column densities of various ions to that of $\mathrm{H}$, with some appropriate ionization correction. However, the results have unavoidable uncertainties due to the uncertain ionization fractions and the fact that the absorption lines are not likely to form all in the same gas. For example, the fraction of the observed $\mathrm{H}$ I column density which arises in the highionization $\mathrm{N}$ V-O VI region is unknown; it depends on the ionizing continuum shape and quite possibly the properties of the outflow, both unknowns. Therefore, instead of attempting to derive abundances independently for each element relative to hydrogen we pursue the more limited goal of constraining the overall metallicity, $Z \equiv \mathrm{Z} / \mathrm{Z}_{\odot}$ (where $\mathrm{X}+\mathrm{Y}+\mathrm{Z} \equiv 1$ ), by assuming the metal abundances either (1) scale uniformly with solar ratios or (2) follow the RSF enrichment scenario of HF93. Specifically, we solve for $Z$ in ionization calculations that are based on two sets of the most abundant ions: $\mathrm{H}_{\mathrm{I}}-\mathrm{C}$ III-N III-O III and $\mathrm{H}$ I$\mathrm{C}$ IV-N v-O vi. These two sets of CNO ions are destroyed on either side of the $\mathrm{He}^{+}$ionization edge. We consider them separately and together to explore one- and two-zone models of the absorbing gas. Adopting ab initio CNO abundance ratios (scaled solar or RSF) has the following advantages. (1) It minimizes the number of free parameters in the ionization calculations. This allows us to solve simultaneously for both $Z$ and the ionization structure in each fully self-consistent calculation. (2) It allows us to test directly the RSF abundance scenario for BAL gas and see what additional constraints that particular CNO behavior can provide for the overall $Z$ derivations.

The RSF chemical enrichment model adopted here is similar to "standard" models of massive elliptical galaxies (Arimoto \& Yoshii 1986; Matteucci \& Greggio 1986; Mat- teucci \& Tornambe 1987; Bica 1988). In these models, nitrogen is mainly a secondary element whose enrichment scales as $\sim Z^{2}$ (see HF93). We will show that this behavior can provide an important additional constraint on the metallicity. Ratios between the column densities of carbon and oxygen have little dependence on the abundances, but they will help set the ionization.

Among the poorly constrained parameters, the most important are the shape of the ionizing spectrum and the possible stratification or inhomogeneity of the BAL region. We quantify the consequent uncertainties by showing explicitly how the results depend on several key parameters. Our approach will be to examine predicted column densities for a variety of conditions in photoionized clouds, using scaled solar and RSF abundances and various continuum shapes, and then to compare them to the BAL column densities of $0226-1024$ measured by K92.

In $\S 2$ we summarize our assumptions regarding the calculations and describe our methods of investigating the metallicity. In $\S 3$ we investigate scaled solar and RSF abundance scenarios. In $\S 4$ we discuss the implications of enhanced abundances in BAL gas due to RSF evolution and summarize the results.

\section{OVERVIEW OF THE CALCULATIONS}

To predict the BAL column densities, we used the spectral synthesis code CLOUDY, version 86.06 (Ferland 1994). We assume that the BAL gas is photoionized by a continuum consistent with observations and can be described by constant particle density, constant ionization parameter, plane-parallel slabs. Justifications and caveats for each of these assumptions follow. Further details regarding these assumptions are found in the Appendices. The major differences between this and previous work are that (1) we test directly the RSF enrichment scenario that appears most appropriate for chemical evolution in massive galactic nuclei, (2) the effects of a large range of ionizing continuum shapes are investigated, and (3) we use column densities of seven ions to constrain the calculations more than previously used in the analysis of BAL QSOs (but see Wampler, Chugai, \& Pettitjean 1995 for their analysis of the unusual Mg II-Fe II BAL QSO 0059 - 2735).

We investigated the coupled problem of the ionization equilibrium and metallicity in three stages by considering (1) the highly ionized gas alone, (2) the doubly ionized metal gas alone, and (3) both types of gas simultaneously in a simple "single-cloud" calculation. We utilize three types of chemical abundances: $(a)$ solar abundances, $(b)$ a simple, but ad hoc, enrichment scenario of scaled solar abundances, and $(c)$ the RSF chemical enrichment model.

\subsection{The Ionizing Continuum \\ 2.1.1. Spectral Energy Distribution}

BAL gas is thought to be photoionized by the central source (WTC85). Thus, knowledge of the spectral energy distribution of the ionizing photons is important. However, since the shape of the ionizing continuum in $0226-1024$ and other BAL QSOs is largely unknown, we investigate the impact of a range of ionizing continuum shapes, reflecting the range in observed QSO continue. (See Appendix A for a discussion of the observational constraints on the shape of the ionizing continuum in this and other BAL QSOs.) We use two components to describe the ionizing 
continuum: (1) A UV bump component described by the following relation:

$$
f_{v} \propto v^{\alpha_{u v}} \exp \left(-h v / k T_{\text {cutoff }}\right) \exp \left(-k T_{\text {low }} / h v\right),
$$

with a high-energy exponential cutoff characterized by the temperature $T_{\text {cutoff }}$ and a low-energy exponential cutoff characterized by $T_{\text {low }}=1000 \mathrm{~K}$. Tests show that the exact value of the low-energy cutoff has little effect on the results as long as it is high enough to prevent unrealistically high IR brightness temperatures that would result in a significant amount of free-free heating at the front face of the cloud (Ferland \& Persson 1989; Ferland et al. 1992). We assume that the IR flux is unimportant in the BAL region because most of the observed IR continuum in QSOs comes from warm dust at large distances from the central source (Sanders et al. 1989; Barvainis 1990). (2) A power-law $\left(f_{v} \propto\right.$ $\left.v^{\alpha_{x}}\right)$ component between the high-energy cutoff of the UV bump and $50 \mathrm{keV}$ of spectral index $\alpha_{\mathrm{x}}=-0.9$, which is thought to be the intrinsic power-law slope of the X-ray continuum before striking the Compton reflector (see Nandra et al. 1991; Smith, Done, \& Pounds 1993). The results are much less sensitive to the actual logarithmic slope of this component than they are to the shape and cutoff energy of the UV bump.

Two logarithmic slopes of the UV bump component, $\alpha_{\mathrm{uv}}$, were chosen to produce different effects in the poorly constrained EUV, in which most of the reprocessing of the radiation occurs. Figure 1 illustrates the two types of UV bump continua, and, for comparison, the mean active galactic nucleus (AGN) continuum inferred by Mathews \& Ferland (1987). The dashed curve has a UV bump with $\alpha_{u v}=0.5$, which matches approximately that of simple accretion disk calculations, although the slope can be a function of the various disk parameters such as inclination (see Madau 1988; Laor \& Netzer 1989; Sun \& Malkan 1989).
We will refer to this as the $v^{0.5} \mathrm{UV}$ bump. The solid curve has an $\alpha_{\mathrm{uv}}=-0.5$, which is the mean value measured longward of $1300 \AA$ in luminous QSOs (see Francis 1993). We will refer to this type as the $v^{-0.5}$ UV bump. Both UV bumps in Figure 1 have the same hydrogen ionizing photon flux, and the values of $T_{\text {cutoff }}$ were chosen such that both continua peak in energy at 1 ryd $\left(\log T_{\text {cutoff }}=5.022\right.$ for the $v^{0.5}$ UV bump and 5.499 for the $v^{-0.5}$ UV bump). We note that the energy at the peak (in $v F_{v}$ ) of the UV bump is related to $T_{\text {cutoff }}$ by

$$
E_{\text {peak }} \approx 8.62\left(1+\alpha_{\mathrm{uv}}\right) T_{5} \mathrm{eV},
$$

where $T_{5}$ is the $T_{\text {cutoff }}$ in units of $10^{5} \mathrm{~K}$. Both continua in this example have the same $\alpha_{o x}(-1.68)$ which characterizes the logarithmic continuum slope between $2500 \AA$ and $2 \mathrm{keV}$ if the UV-X-ray continuum could be described as a single power law (Zamorani et al. 1981). Note that we prefer an explicit minus sign in the definition of $\alpha_{o x}$. Once the choice of $\alpha_{u v}$ is made for the shape of the UV bump, the two parameters, $T_{\text {cutoff }}$ and $\alpha_{o x}$, describe the shape of the ionizing continuum (see also Ferland et al. 1996), and we will explore the full observed range of these two parameters that describe QSO continua.

The shape of the continuum at energies $<1$ ryd is not important. We use the two values of $\alpha_{\mathrm{uv}}$ to dramatize the effects of different slopes across the important but observationally unconstrained region between $\sim 10 \mathrm{eV}$ and 0.1 $\mathrm{keV}$. The $v^{0.5} \mathrm{UV}$ bump drops more sharply than does the $v^{-0.5} \mathrm{UV}$ bump, such that in this example, the $\mathrm{N} \mathrm{v}$ ion is created and destroyed on the extreme tail of the UV bump, while the $\mathrm{O}$ VI ion is created and destroyed on the X-ray power-law component. Conversely, the $v^{-0.5}$ UV bump drops more gradually, such that both these ions are created and destroyed on the bump. One obvious effect is that cal-

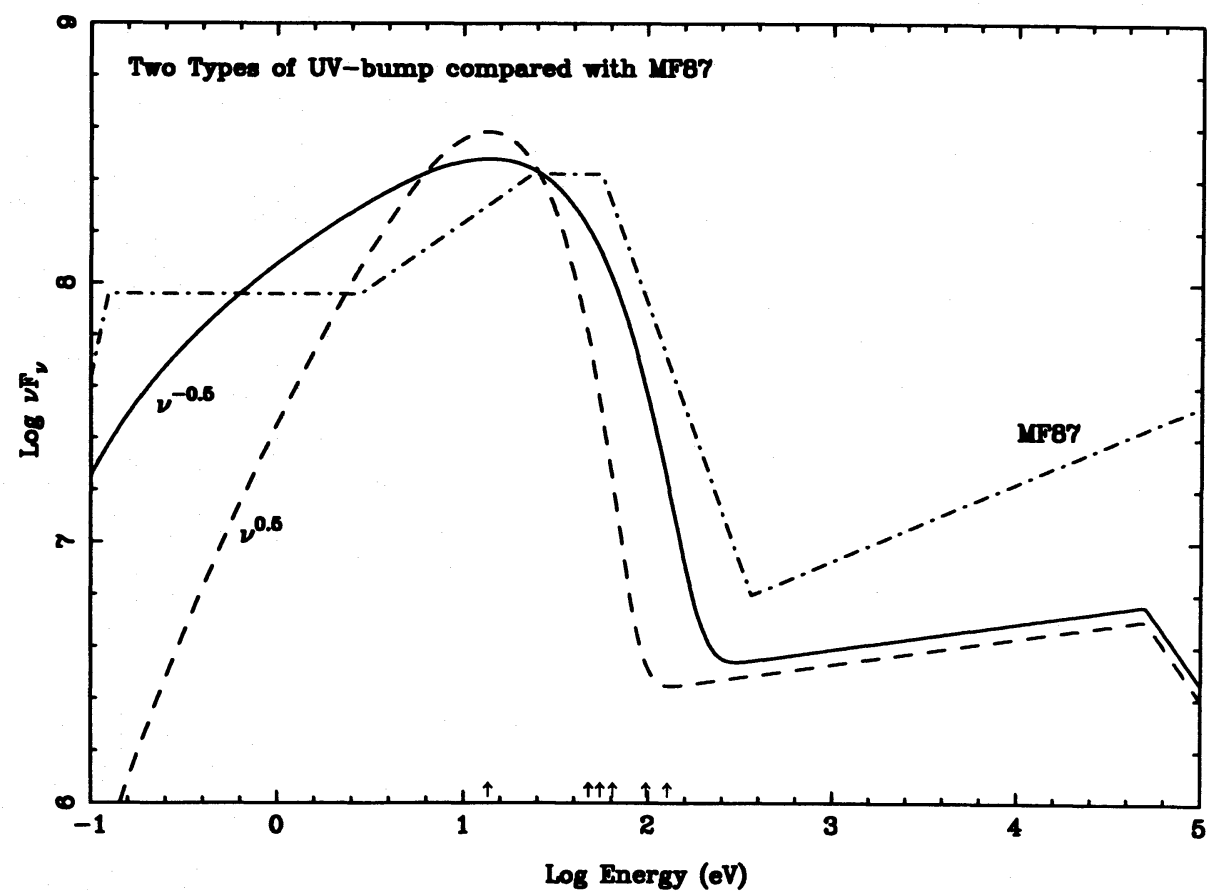

Fig. 1.-An illustration of the two types of UV bump continua used. The dashed continuum has an $f_{v} \propto v^{0.5}$ UV bump, while the solid continuum has an $f_{v} \propto v^{-0.5}$ UV bump. The hydrogen ionizing flux in each continuum is the same, and both have an $\alpha_{o x}$ of -1.68 . The important differences arise mainly in the shapes of their UV bump peaks and exponential tails. For comparison, the MF87 continuum with the same hydrogen ionizing flux is represented by the dot-dashed curve. The ionization energies of $\mathrm{H}_{\mathrm{I}}, \mathrm{C}$ III and $\mathrm{N}$ III, $\mathrm{O}$ III, $\mathrm{C} \mathrm{IV}, \mathrm{N} \mathrm{v}$, and $\mathrm{O}$ vi, respectively, are marked with arrows along the bottom. 
culations which use the $v^{0.5}$ UV bump will be much more sensitive to $T_{\text {cutoff }}$ than those which use the $v^{-0.5} \mathrm{UV}$ bump.

\subsubsection{The Ionization Parameter}

In these calculations, the intensity of the ionizing photons from the UV bump was set with the dimensionless ionization parameter, which we define here as

$$
U_{\text {bump }}=\frac{\Phi_{\text {bump }}}{n(\mathrm{H}) c},
$$

where $\Phi_{\text {bump }}$ is the flux of the UV bump hydrogen ionizing photons incident at the front face of the cloud, $n(\mathrm{H})$ is the total hydrogen number density, and $c$ is the speed of light. Note that the total ionization parameter may be slightly larger than this value, depending on the value of $\alpha_{o x}$ that normalizes the X-ray power law to the UV bump component. Although it is important to the predicted emissionline spectrum, the choice of particle density $\left(10^{9} \mathrm{~cm}^{-3}\right)$ has only minor effects on the ionization equilibrium and derived column densities. Each numerical simulation of the BAL gas assumed a single ionization parameter planeparallel slab. The generally good fits of the C IV BAL template to the other BAL troughs of $0226-1024$, over a wide range in ionization ( $\mathrm{C}$ III to $\mathrm{O} \mathrm{VI}$ ), argues that the ionization parameter cannot change drastically over the observable radial velocity extent of the BAL flow (K92). Note, however, that the assumption of approximately constant ionization parameter with the flow speed does not exclude the possibility that the lower ionization BALs form in regions of high density that are flowing with, or producing, via ablation, the lower density gas that would manifest itself in the $\mathrm{N} v$ and O vi BALs (see Voit et al. 1993). In this way, two separate regimes in ionization may coexist within the outflow.

\subsection{The Observed Column Densities: Constraints}

We use the column densities measured by K92 for the BAL QSO $0226-1024$ to constrain the calculations. Table 1 shows the values and estimated internal uncertainties of the measured integrated column densities of $\mathrm{H}$ I and various $\mathrm{C}, \mathrm{N}$, and $\mathrm{O}$ ions. These uncertainties are subjective and meant to reflect the quality of the fit, given the assumptions made in K92. (See Appendix B for a discussion concerning the assumptions K92 made when determining column densities from the observed BAL troughs.) Hereafter, we designate the column density $\left(\mathrm{cm}^{-2}\right)$ of an ion $X$ as $N(X)$. We emphasize that $N(\mathrm{H} \mathrm{I}), N(\mathrm{C}$ IV $), N(\mathrm{~N}$ v), and $N(\mathrm{O} \mathrm{VI})$ in Table 1 are typical for BAL QSOs. [See WTC85 and Hamann et al. (1993) for mean BAL column densities measured from ground-based spectra, although the mean

TABLE 1

Column Density Measurements OF $0226-1024$

\begin{tabular}{cc}
\hline \hline Ion & $\begin{array}{c}\log \text { of } \\
\text { Column Density } \\
\left(\mathrm{cm}^{-2}\right)\end{array}$ \\
\hline $\mathrm{H}_{\text {I }} \ldots \ldots \ldots \ldots$ & $15.8 \pm 0.1$ \\
$\mathrm{C}$ III $\ldots \ldots \ldots \ldots$ & $15.6 \pm 0.2$ \\
$\mathrm{C}$ IV $\ldots \ldots \ldots \ldots$ & $16.20 \pm 0.05$ \\
$\mathrm{~N}$ III $\ldots \ldots \ldots \ldots$ & $16.3 \pm 0.2$ \\
$\mathrm{~N}$ v $\ldots \ldots \ldots \ldots$ & $16.3 \pm 0.1$ \\
O III $\ldots \ldots \ldots \ldots$ & $16.7 \pm 0.2$ \\
O VI $\ldots \ldots \ldots \ldots$ & $16.5 \pm 0.1$ \\
\hline
\end{tabular}

$N(\mathrm{O}$ vI) estimate in WTC85 is very uncertain because few measurements were available at that time.] We do not include K92's measurements of Si IV $\lambda \lambda 1393,1403$ or any of the sulfur ions in this analysis, since their recombination coefficients are not well known. Nor do we include the measurements of $N(\mathrm{~N}$ IV) or $N(\mathrm{O}$ IV $)$, as they were deemed too uncertain. We note that the lower ionization column densities in Table 1 may suffer more from the systematic errors discussed in Appendix B than do those of $\mathrm{C} \mathrm{IV}, \mathrm{N}$ v, and $\mathrm{O}$ $\mathrm{VI}$; note also that their internal uncertainties are larger.

Similar to the broad emission-line spectrum of QSOs, it has always been difficult to explain the observed broad range in BAL gas ionization if it occurs in a singleionization parameter "cloud" (see WTC85). In much of the analysis which follows, we will address the questions of ionization equilibrium and metallicity in three steps. In step 1, we will consider only the three high-ionization BALs, C IV, $\mathrm{N} v$, and $\mathrm{O}$ vi, using the measured $N(\mathrm{H} \mathrm{I})$ as an upper limit. For these calculations we will assume that the total measured $N(\mathrm{C}$ IV $)$ lies within the highly ionized gas, even if this is not likely to be the case. For a fixed $N(\mathrm{O} \mathrm{VI})$, a larger $N(\mathrm{C}$ Iv) will result in a larger $N(\mathrm{~N} \mathrm{v})$ until the latter is fully formed within the cloud. By assuming the maximum amount of $\mathrm{C}$ IV present, we will maximize the predicted $N(\mathrm{~N}$ v) and thus minimize the predicted metallicity. In step 2, we will consider only the three doubly ionized metal BALs, C III, $\mathrm{N}$ III, and $\mathrm{O}$ III, using the measured $N(\mathrm{H}$ I) and $N(\mathrm{C}$ IV $)$ as upper limits. Only part of measured $N(\mathrm{H} \mathrm{I})$ can coexist with either the high-ionization metal line gas or the doubly ionized metal gas, so we want the calculations in steps 1 and 2 to underpredict $N(\mathrm{H} \mathrm{I})$. It is also true that a substantial fraction of C IV is likely to form in the region which forms the doubly ionized metal BALs, while the latter are not expected to form in the region which forms the highest ionization species (e.g., $\mathrm{N} v$ and $\mathrm{O}$ vI). Thus, the $N(\mathrm{C}$ IV) will constrain the calculations in step 2 as an upper limit. In step 3 we will consider all seven column densities listed in Table 1 together in a single "cloud." While the last step might appear advantageous for better constraining the ionization equilibrium and thus the metallicity, it is possible that the two types of gas are not present in "clouds" of the same ionization parameter $(\$ 2.1 .2)$. Calculations which include multidensity gas condensations within the flow are beyond the scope of the present investigation.

\subsection{The Optimization Grids}

To understand the impact of unknown or ill-constrained parameters, we have examined grids of optimized calculations that search for the best match to the measured column densities. For example, in $\S 3.3 .1$ we will examine a range of ionizing continuum shape parameters, $T_{\text {cutoff }}$ and $\alpha_{o x}$. For each choice of ionizing continuum shape, we solve simultaneously for three other parameters: the UV bump ionization parameter, $U_{\text {bump }}$, the metallicity, $Z \equiv \mathrm{Z} / \mathrm{Z}_{\odot}$, and the total hydrogen column density, $N(\mathrm{H})$, while optimizing the match to the measured BAL column densities. Here $\mathrm{Z}$ is the mass fraction metal abundance in the relation $\mathrm{X}+\mathrm{Y}+\mathrm{Z} \equiv \mathbf{1}$.

Two separate optimizing routines were utilized: the AMOEBA "down-hill simplex" (Press et al. 1992) and the SUBPLEX method (Rowan 1990). SUBPLEX is a subspace searching simplex method similar to the "down-hill simplex" of AMOEBA and is somewhat more robust than AMOEBA. Both methods gave similar results, although the 
SUBPLEX routine generally found somewhat deeper minima. We experimented with different initial parameter guesses, parameter step sizes, and uncertainties of the constraining column densities for the optimization runs. We found that the optimizer was generally robust enough to find very similar minima for different initial conditions. This will generally be the case as long as the $\chi^{2}$ manifold is smoothly varying with a single deep minimum. The other extreme is a manifold which appears globally like the weakly dimpled surface of a golf ball; the solution found is then heavily weighted by the initial starting conditions. In this extreme case, the contouring of parameters, such as the metallicity, upon the continuum shape plane becomes impossible. In the contours plots we show below, however, this effect will manifest itself only locally as "noise" on the contours. In this case, the manifold is dimpled locally near the absolute minimum because the functional dependence of the optimized parameter on the continuum shape becomes small. The subplex does not always find this absolute minimum and chooses one nearby instead, producing small kinks and peninsulas on what otherwise would be smooth contours. Nevertheless, nearly all the optimized fits below matched the measured column densities constraining them to the precision given in Table 1 , and in no case did the predicted column densities differ from the observed value by more than $1 \sigma$.

\section{RESULTS OF CALCULATIONS}

\subsection{Solar Abundances}

Before we explore whether the measured column densities might imply enriched metal abundances, we determine first whether some ionizing continua can reproduce the measured highly ionized column densities with a solar metallicity and solar relative abundances (from Grevesse \& Anders 1989). We will show that, except for a very narrow range in continuum shapes, solar abundances cannot match simultaneously the observed column densities of $\mathrm{C}$ IV, $\mathrm{N} \mathrm{v}$, and $\mathrm{O}$ VI.

First we ran photoionization calculations to fit the measured $N\left(\mathrm{C}\right.$ Iv), $N(\mathrm{~N} \mathrm{v})$, and $N\left(\mathrm{O}\right.$ vi) (Table 1) with $T_{\text {cutoff }}, \alpha_{o x}$, and $U_{\text {bump }}$ allowed to vary as free parameters. For each calculation we adopted a value of $\log N(\mathrm{H} \mathrm{I})$, from $10 \%-$ $100 \%$ of the measured value. We show the results schematically in Figures $2 a$ and $2 b$ for the $v^{0.5}$ and $v^{-0.5} \mathrm{UV}$ bumps, respectively. The solid diagonal line represents solutions which match exactly the measured values of all three column densities for differing choices of the neutral hydrogen column density, as marked along the diagonal line. Solutions in the $\alpha_{o x}-\log T_{\text {cutoff }}$ plane which could match the measured $\mathrm{C}$ IV and $\mathrm{O}$ VI column densities exist to the left of the solid diagonal lines in each figure, but they predict too little $\mathrm{N} \mathrm{v}$, by up to factors of $2-3$. The dot-dashed and dashed lines in each figure have $\mathrm{N} v$ logarithmic column densities of 16.0 and 16.15 , respectively, compared to the measured value of $16.3 \pm 0.1$. As can be seen from Figures $2 a$ and $2 b$ solutions for a given $N(\mathrm{H} \mathrm{I})$ which match the $\mathrm{C}$ IV and $\mathrm{O}$ VI column densities move from the upper left toward the lower right, one for each value of the $N(\mathrm{H} \mathrm{I})$, terminating at the solid diagonal line. To the right of the solid diagonal line, either no solutions exist matching $N(\mathrm{C} \mathrm{IV})$ and $N(\mathrm{O} \mathrm{VI})$, or $N(\mathrm{~N} v)$ is overpredicted by factors of $\sim 2$. Nowhere on the continuum shape plane do the predicted doubly ionized metal column densities approach their measured values. It is also noted that we could not find good fits with solar abundances using the doubly ionized metal column densities as constraints instead of the high-ionization ones.

The results in Figures $2 a$ and $2 b$ reveal some problems with the assumption of solar metallicity and solar relative abundances. First, rather soft UV bumps are implied for solar abundances. As constrained by the measured $N(\mathrm{H} \mathrm{I})$ $\left(10^{15.8} \mathrm{~cm}^{-2}\right)$, the $v^{0.5}$ and the $v^{-0.5} \mathrm{UV}$ bumps peak at energies $E_{\text {peak }}=21.5 \mathrm{eV}$ and $11.3 \mathrm{eV}$, marginally consistent with the observation of 0226-1024 (see Appendix A). However, when we consider that a substantial portion of the measured $N(\mathrm{H} \mathrm{I})$ also must exist in the region of doubly ionized metals, the predicted UV bumps become still softer. Weak incident X-ray continua are also predicted, although BAL QSOs may have weaker than normal X-ray fluxes (see Appendix A). In addition, if, as we expect, a substantial portion of $N(\mathrm{C}$ IV $)$ exists in the region of doubly ionized metals, the curves in Figures $2 a$ and $2 b$ move in a direction perpendicular to themselves toward the lower right in the figures, which increases the area of parameter space for which the $\mathrm{N} v$ column density is underpredicted for solar abundances.

These difficulties, and the lack of good fits to the lowionization column densities for any continuum shape, suggest strongly that strictly solar abundances are not viable.

\subsection{Metal Abundances Scaled from Solar}

Here we explore calculations in which all the metal abundances are scaled from solar by a single constant. Although not realistic from a chemical evolution standpoint, this scenario has the advantage of simplicity. The strength of the metals relative to neutral hydrogen will constrain the metallicity, making this approach not so very different from the traditional metal/H I method. We will show that, except for a very narrow range in continuum shapes, scaled solar abundances that match the measured $N(\mathrm{C}$ IV $)$ and $N(\mathrm{O}$ vI $)$ do not predict enough $\mathrm{N} v$. We will also demonstrate the limited use of scaled solar abundances to predict the gas metallicity.

We ran scaled solar abundance photoionization calculations in which the UV bump ionization parameter $U_{\text {bump }}$, $\alpha_{o x}$, and $\log T_{\text {cutoff }}$ were all free to vary to match the measured C IV, N V, and O VI column densities of $0226-1024$. The logarithmically scaled metallicities investigated were $\pm 0.3, \pm 0.6$, and \pm 0.9 . For each of these scaled metallicities, we ran 11 calculations, one for each adopted value of $\log N(\mathrm{H}$ I) from 14.8 to 15.8 . The method and results, illustrated in Figure $2 c$, are completely analogous to those presented in Figure $2 a$. Here we will discuss the results for the $v^{0.5}$ UV bump only; those for the $v^{-0.5}$ UV bump differ only in detail.

The solid curve in the center of Figure $2 c$ is the same as shown in Figure $2 a$ (i.e., strictly solar abundances). Progressing from lowest to highest in metallicity are sequences of symbols which lie along the solar metallicity solution. The lower values of $N(\mathrm{H}$ I $)$ lie toward the bottom of each metallicity sequence. The low-metallicity solutions lie in the soft UV bump, steep $\alpha_{o x}$ (lower left) portion of the diagram. The other freely varying parameter, $U_{\text {bump }}$, increases in the direction of lower $N(\mathrm{H}$ I) within a sequence, and in the direction of lower metallicity among the sequences. The total hydrogen column density also increases in the direction of lower metallicity. These effects are expected because 


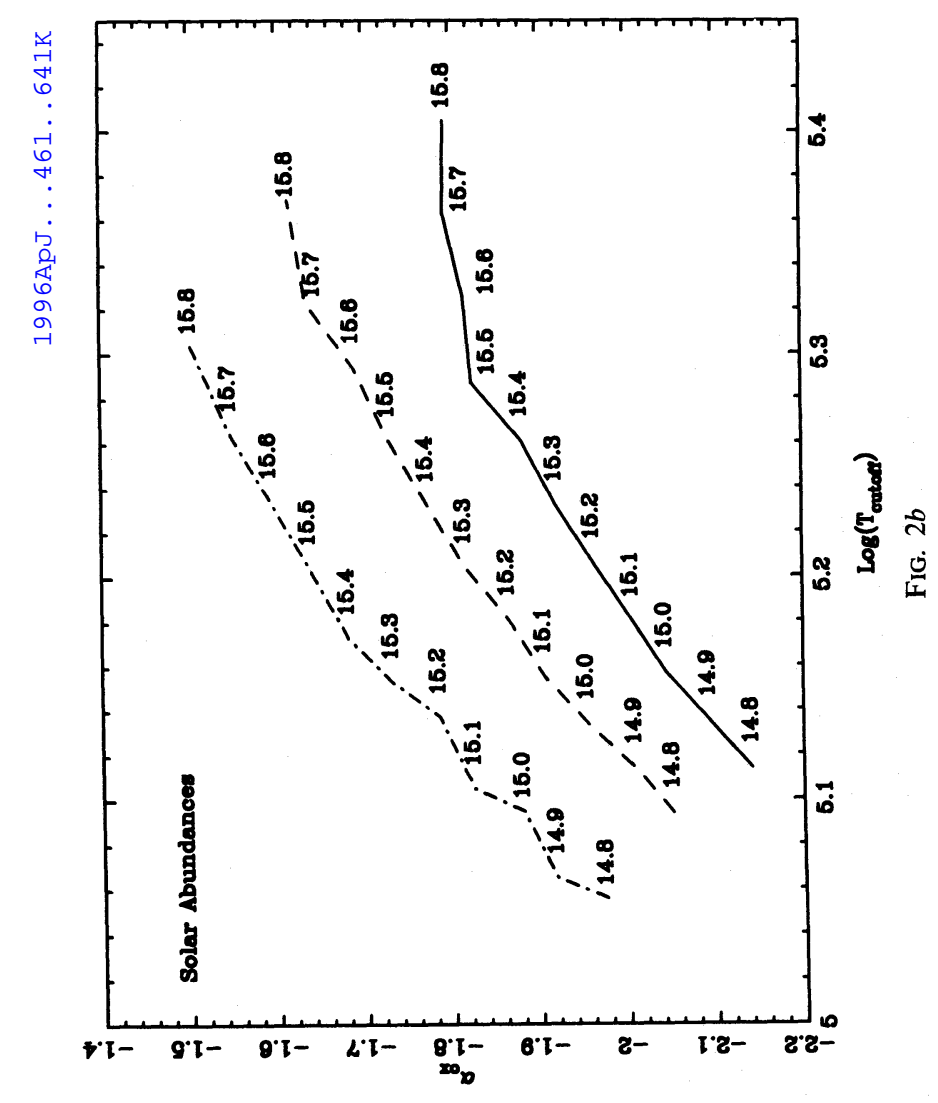

Z 萬定

ह

5 \&

일

ฉ0

ช

T.

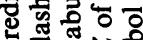

要架

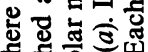

3 .

के के

承焉

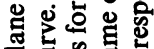

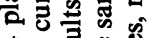

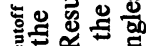

으워

Tें

$\checkmark$.

空零

为告要

可

징유

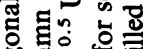
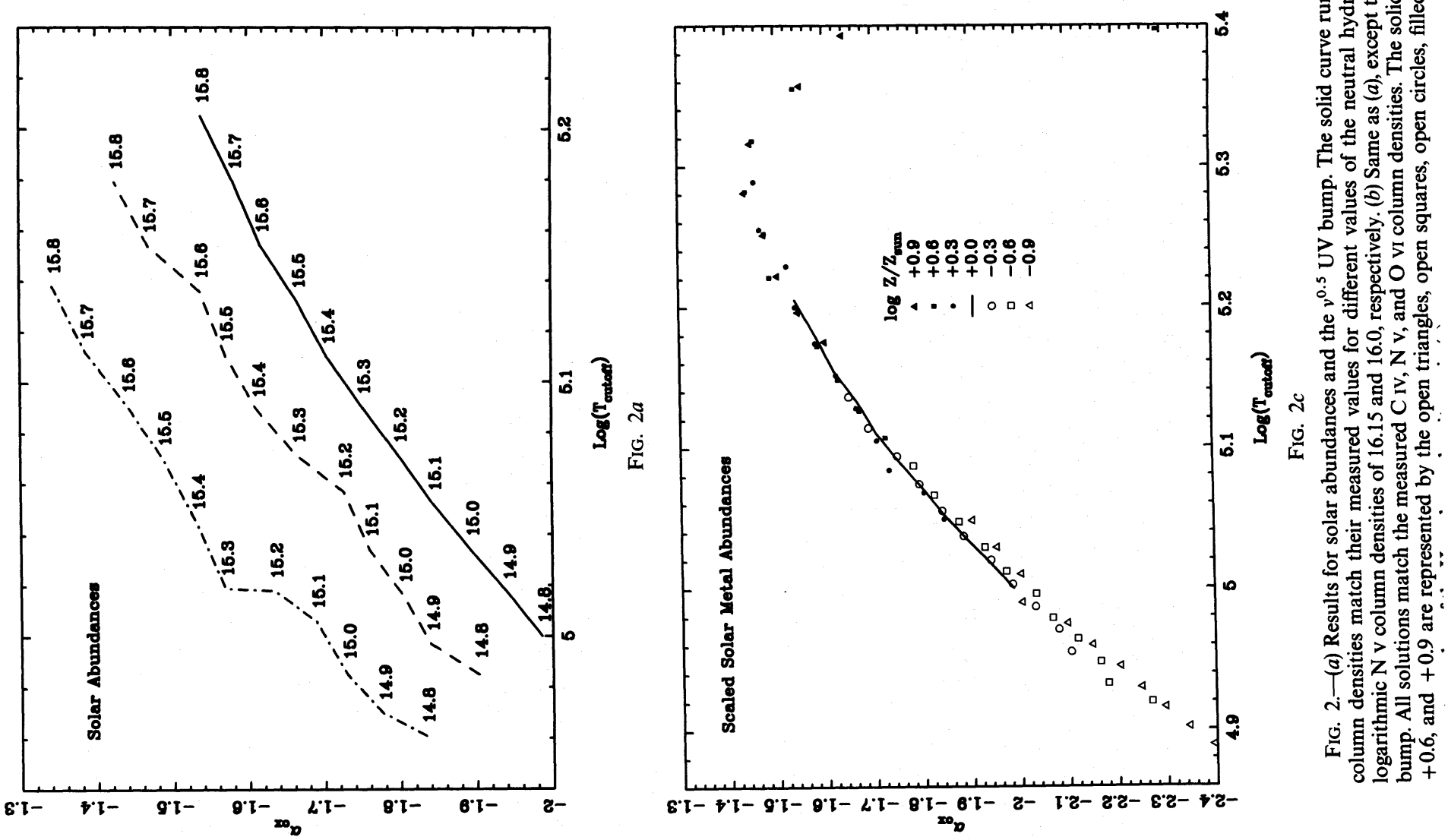
as the metallicity is decreased, larger total hydrogen column densities are required to produce the same metal column densities, and larger ionization parameters are required to ionize the correspondingly more optically thick material. Therefore, the solution for scaled solar metallicity will simply slide along the solar curve from Figure $2 a$. This simple relationship begins to break down, however, for large $N(\mathrm{H}$ I $)$ in high-metallicity clouds because (1) the metals are beginning to rival helium as the primary continuous opacity source, (2) the peak in the UV bump is moving to energies which put the ionizing photons of hydrogen and the highly ionized metals on distinctly opposite sides. The point representing the largest $\log N(\mathrm{H}$ I) (15.8) for $\log Z=0.6$ in the lower right corner is not reliable, and the largest four $N(\mathrm{H} \mathrm{I})$ values for $\log Z=0.9$ are not shown because their dependencies on $\alpha_{o x}$ have become weak as the bump takes over as the agent of ionization for these ions. The quality of these fits also tended to be low. Metallicities larger than $\log Z=0.9$ are possible, but ever smaller $N(\mathrm{H}$ I $)$ will be required, as the solutions for the larger $N(\mathrm{H} \mathrm{I})$ "fall off" the curve.

Note that for most values of $\alpha_{o x}$ and $\log T_{\text {cutoff }}$ which are consistent with observations of non-BAL QSOs (Appendix A), the predicted metallicities are solar and larger. The lower limit of solar metallicity derives from the magnitude of the metallic column densities compared to the maximum $N(\mathrm{H} \mathrm{I})$. Just as in Figures $2 a$ and $2 b$, there are solutions to the left of the curve in Figure $2 c$ which match the measured $N(\mathrm{C}$ IV $)$ and $N(\mathrm{O}$ vI) but underpredict the $N(\mathrm{~N}$ v). Also, as mentioned above, we expect a significant fraction of the measured $N(\mathrm{C}$ Iv $)$ to originate in a region outside that of the high-ionization gas. Smaller $N(\mathrm{C}$ Iv) in the high-ionization gas would move the curve in Figure $2 c$ to the lower right, increasing the area in the continuum shape plane for which the $N(\mathrm{~N} v)$ is underpredicted relative to $N(\mathrm{C}$ IV $)$ and $N(\mathrm{O}$ vi).

Clearly, scaled solar abundances provide limited constraints on the overall metallicity, apart from the firm lower limit of $Z>Z_{\odot}$. A simultaneous match to the $\mathrm{C}$ IV, $\mathrm{N} \mathrm{v}$, and $O \mathrm{VI}$ column densities requires a rather narrow range in ionizing continuum shapes, and solutions of differing metallicities overlap on the continuum-shape plane. As in the case of strictly solar abundances, satisfactory fits to the doubly ionized metal column densities could not be found.

\subsection{Abundances from Enrichment Calculations}

In the RSF enrichment models, the relative abundance of nitrogen is directly related to the global metallicity. In particular, the $\mathrm{N} / \mathrm{H}$ abundance ratio varies roughly as the square of the metallicity, and $(\mathrm{N} / \mathrm{C})$ and $(\mathrm{N} / \mathrm{O})$ ratios greater than solar occur for several times solar metallicities (HF93). The nearly quadratic buildup of nitrogen occurs because the nitrogen enrichment is dominated by secondary CNO nucleosynthesis in the chemical evolution scenarios adopted here (see HF93). Thus, the column density in nitrogen compared to carbon and oxygen can provide another constraint on the overall metallicity, in addition to the usual constraint provided by the combined metals relative to $\mathrm{H}$ I. The fact that the solar and scaled solar abundance scenarios ( $\$ 3.1$ and 3.2) often failed because they underpredicted the nitrogen column density suggests that we will find improved fits using RSF abundances at supersolar metallicities.

The RSF abundances, while uncertain in detail, have the advantage of being derived from the best available calcu- lations for the enrichment in massive galactic nuclei (see HF93). Other advantages of adopting this approach are discussed in $\S 1$. We adopt the abundance grid "M5a" from HF93, the same as used by Baldwin et al. (1996) and Ferland et al. (1996). Figure 2 in HF93 plots these and other relative abundances as a function of metallicity. In Table 2 we show the $\mathrm{He}$ and $\mathrm{CNO}$ abundances relative to hydrogen and normalized to solar, for values of $\log Z$ ranging from 0 to 1 . Note that $Z=1$ does not imply that the abundances have solar ratios. The relative abundances in this model are identical to the HF93 standard giant elliptical model (M4a) for $\mathrm{Z} \lesssim 10 \mathrm{Z}_{\odot}$, but a truncated IMF allows the gas to reach much higher metallicities before it is locked up in low-mass stars and stellar remnants.

We note that the nitrogen yields in these RSFs calculations are within $20 \%$ of maximum (i.e., complete secondary conversion of the carbon and oxygen into nitrogen in massive stellar envelopes; see HF93). Increasing the nitrogen abundance by this amount would have no effect on the results, but lowering the nitrogen yields by factors up to a few would lead to larger derived values of metallicities, by roughly the same factor. We note also that if the metallicities associated with the $\mathrm{CNO}$ abundance ratios do differ from those given by the RSF enrichment scenario (see Table 2 ), then the calculated $N(\mathrm{H} \mathrm{I})$ will scale inversely with this correction to the metallicity. We will discuss the implications of this.

\subsubsection{Constraints on Z from the High-Ionization Column Densities}

We ran photoionization calculations and optimized fits to the measured $\mathrm{C}$ IV, $\mathrm{N} \mathrm{v}$, and $\mathrm{O}$ VI column densities of $0226-1024$ (see Table 1) by varying the global metallicity, $Z$, the total hydrogen column density, $N(\mathrm{H})$, and the UV bump ionization parameter, $U_{\text {bump }}$. These three parameters were varied simultaneously, and a solution was found for every pair of parameters describing the continuum shape, $\alpha_{o x}$ and $\log T_{\text {cutoff }}$. We used the total measured $N(\mathrm{H} \mathrm{I})$ as an upper limit to constrain the optimization further, because some or most of the neutral hydrogen is likely to reside in less ionized regions. Figures $3 a-3 c$ show the values of $\log Z$, $\log U_{\text {bump }}$, and $\log N(\mathrm{H})$ derived assuming the RSF abundances for the $v^{0.5}$ UV bump. Continuum shapes lying outside the limits shown in Figure 3 were also investigated, but most solutions involving $\alpha_{o x} \gtrsim-1.2$ and $\log T_{\text {cutoff }} \gtrsim 5.2$ produced significantly more $N(\mathrm{H}$ I $)$ than is measured in $0226-1024$. In addition, the quality of the fits to the CNO column densities began to deteriorate just beyond these boundaries, especially in the direction of higher $\log T_{\text {cutoff }}$.

TABLE 2

RSF Abundances Relative to Solar ${ }^{a}$

\begin{tabular}{ccccc}
\hline \hline $\log Z$ & Helium & Carbon & Nitrogen & Oxygen \\
\hline $0.0 \ldots \ldots$ & 0.878 & 0.349 & 0.266 & 1.349 \\
$0.1 \ldots \ldots$ & 0.899 & 0.449 & 0.450 & 1.702 \\
$0.2 \ldots \ldots$ & 0.927 & 0.579 & 0.766 & 2.147 \\
$0.3 \ldots \ldots$ & 0.963 & 0.751 & 1.268 & 2.711 \\
$0.4 \ldots \ldots$ & 1.010 & 0.980 & 2.126 & 3.427 \\
$0.5 \ldots \ldots$ & 1.072 & 1.287 & 3.576 & 4.338 \\
$0.6 \ldots \ldots$ & 1.155 & 1.740 & 6.055 & 5.506 \\
$0.7 \ldots \ldots$ & 1.268 & 2.277 & 10.36 & 7.013 \\
$0.8 \ldots \ldots$ & 1.426 & 3.072 & 17.96 & 8.979 \\
$0.9 \ldots \ldots$ & 1.651 & 4.190 & 31.76 & 11.60 \\
$1.0 \ldots \ldots$ & 1.983 & 5.802 & 57.64 & 15.18 \\
\hline
\end{tabular}

a $\mathrm{Ratios} X / \mathrm{H}$ relative to solar. 


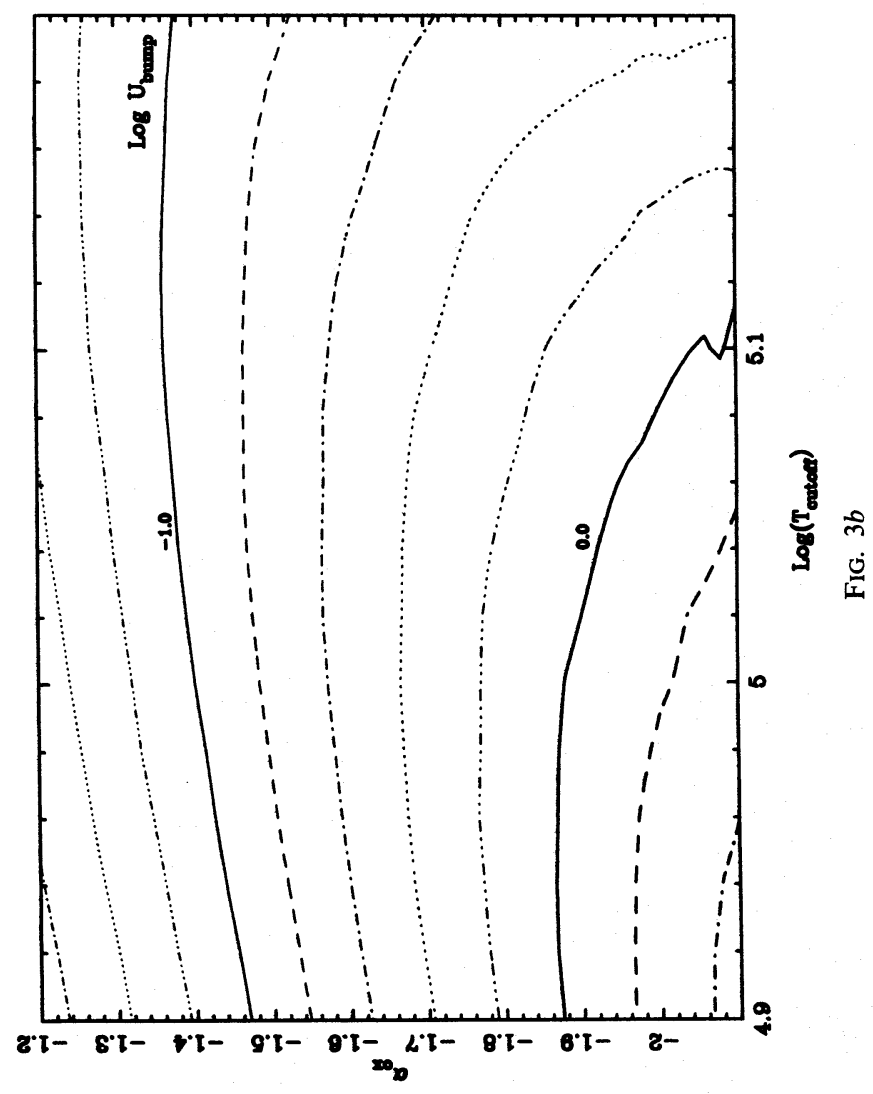

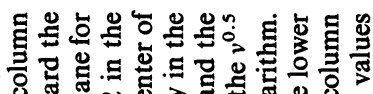
8

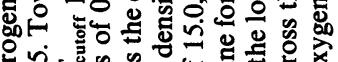

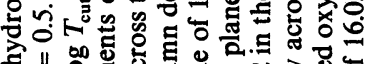

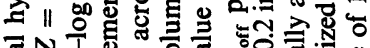

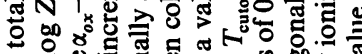

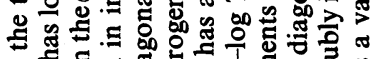

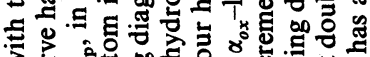

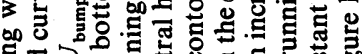

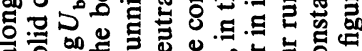
o

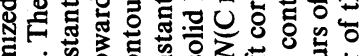
幽

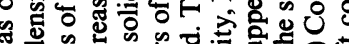
3

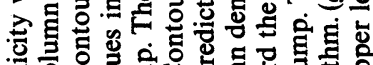

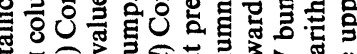

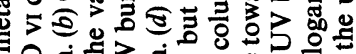

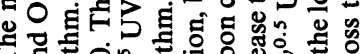
क

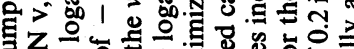
z

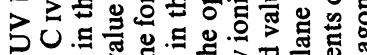

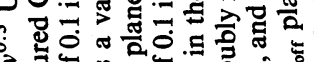

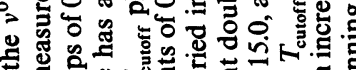

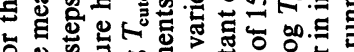

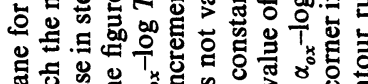

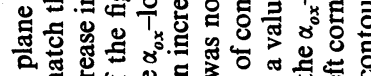

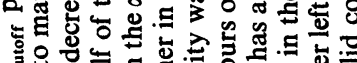

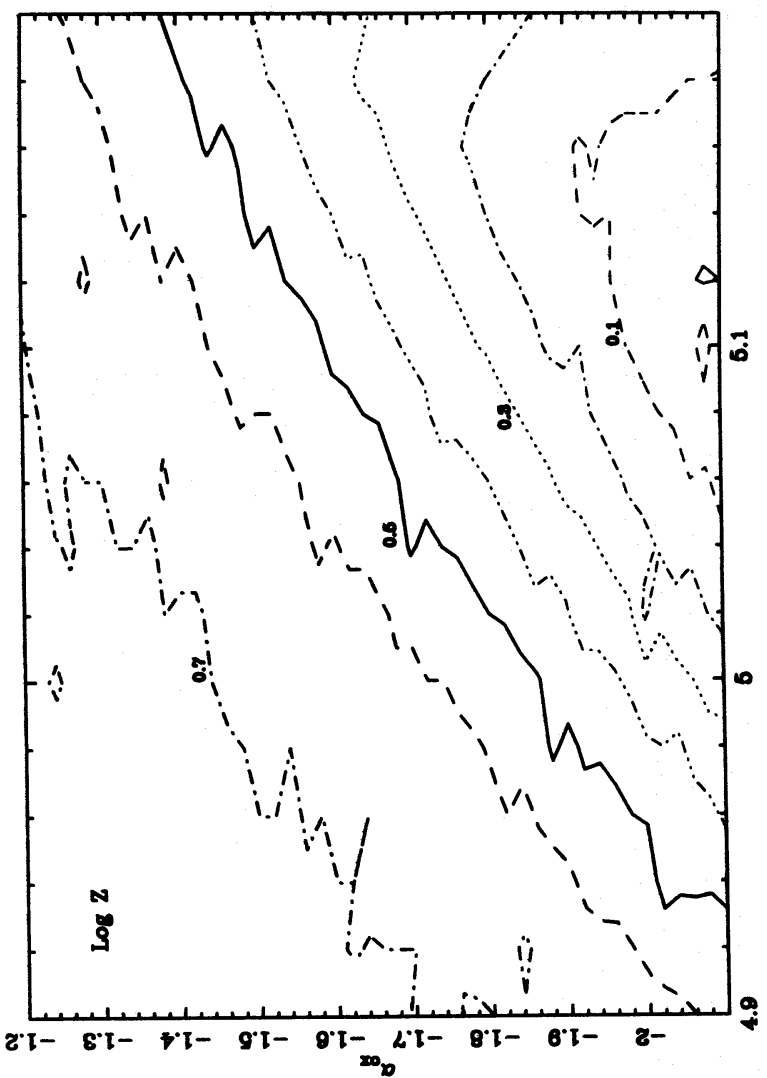

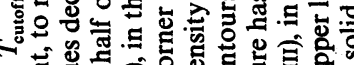
on

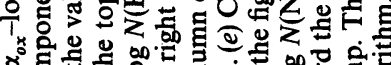

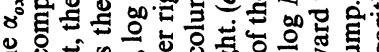
论

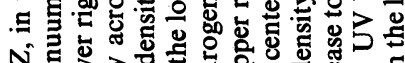
N.

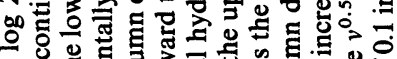

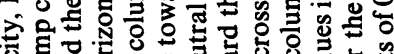

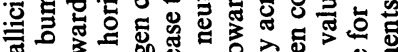
₹

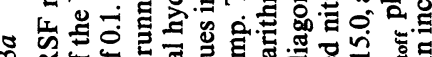
(

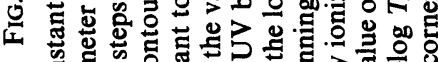

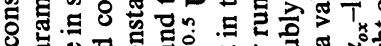

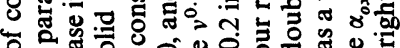

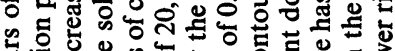
它边.

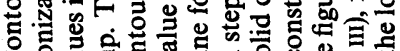

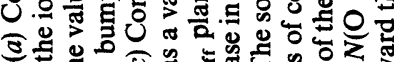

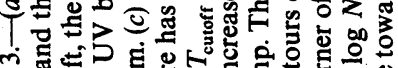

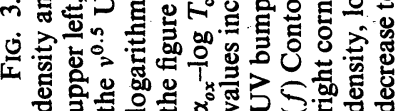



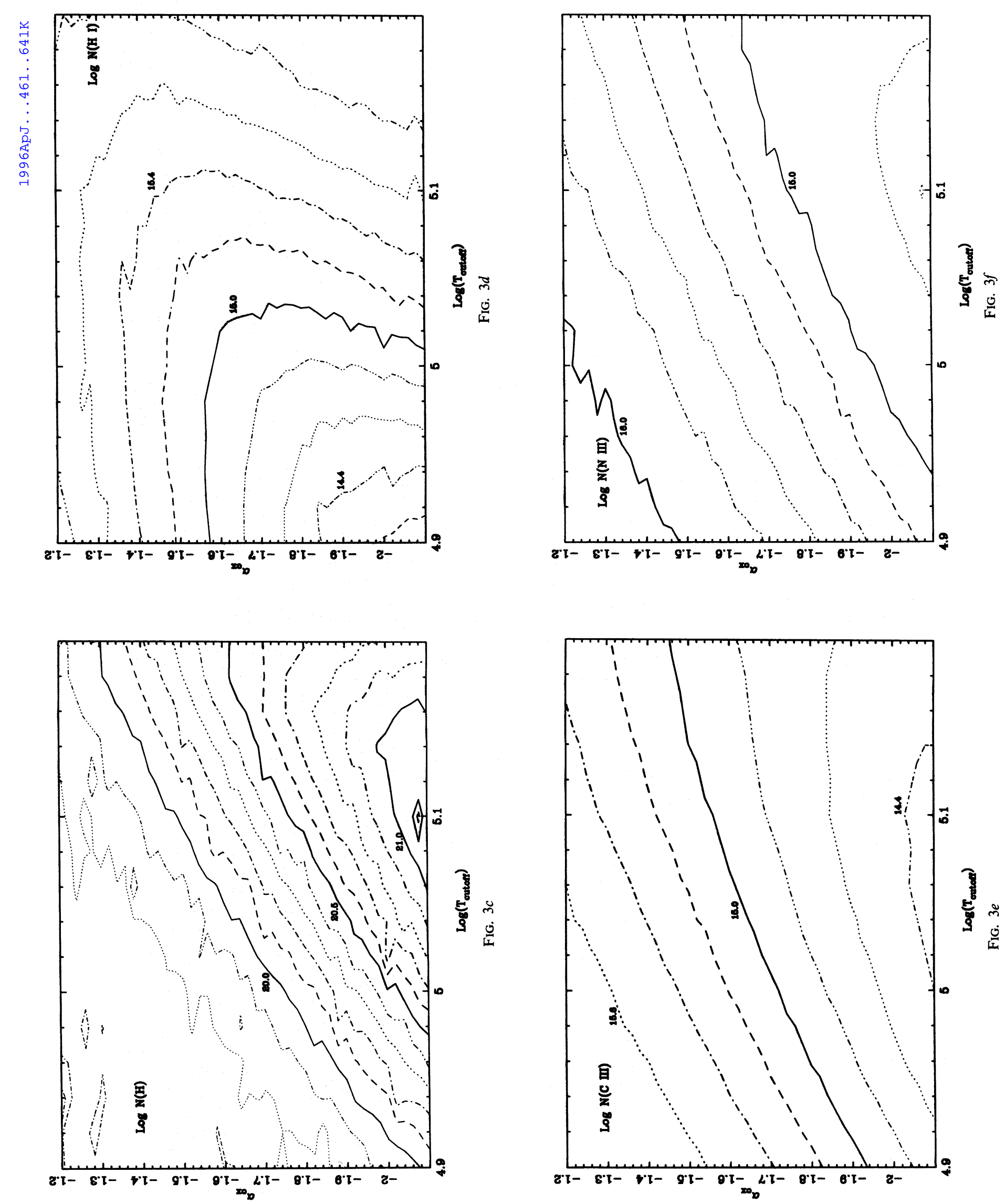


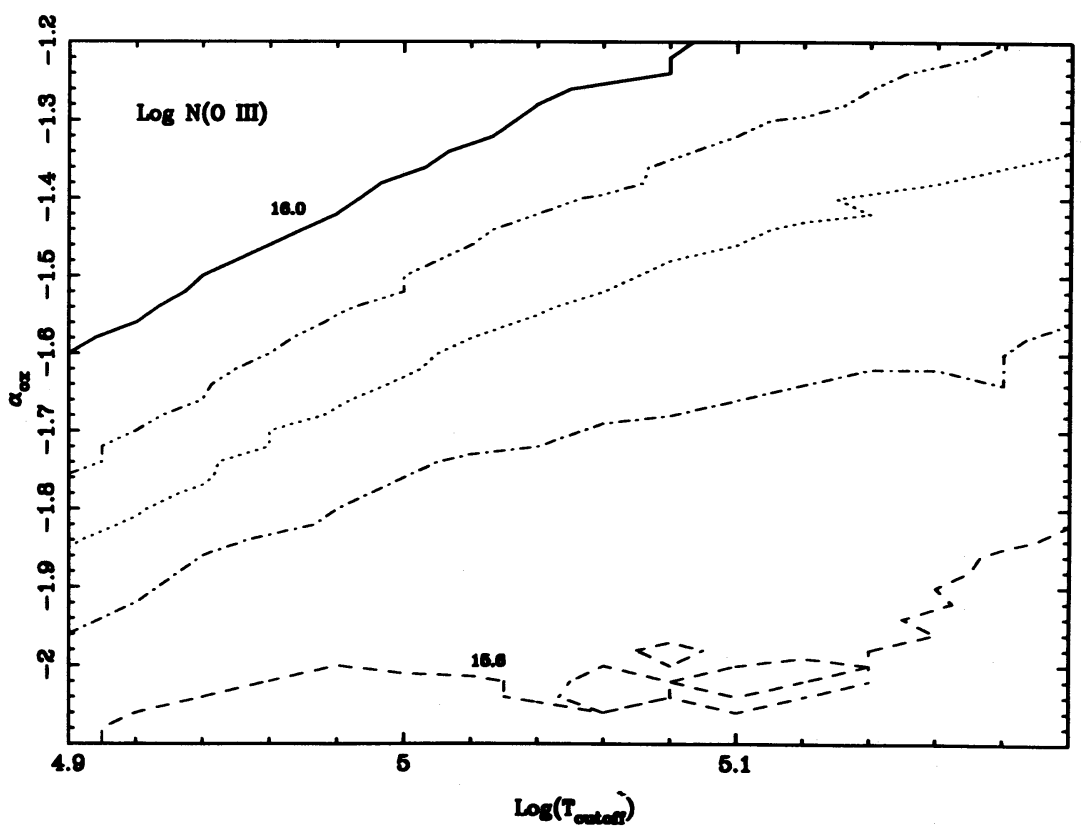

Fig. $3 g$

In all cases, the fits broke down in the region of parameter space outside the contour marking the measured value of $\log N(\mathrm{H} \mathrm{I})(\sim 15.8)$. Also, the calculations tended to become nearly independent of $\alpha_{o x}$ for values significantly less than -2.1 , so those results are not plotted.

The predicted metallicities are contoured in the continuum-shape plane in Figure $3 a$. The solid contour running diagonally across the figure is $\log Z=0.5$. Contours to the right (left) represent lower (higher) $\log Z$ values in steps of 0.1 . For completeness, we show the results of the other two parameters which were varied, $\log U_{\text {bump }}$ and $\log N(\mathrm{H})$, in Figures $3 b$ and $3 c$. The calculations were not constrained to fit the measured neutral hydrogen column density, but values of $\log N(\mathrm{H} \mathrm{I})$ were predicted in each case. In Figure $3 d$ we show its contours on the same ionizing continuum shape-RSF metallicity grid. A comparison of Figures $3 a$ and $3 d$ shows that the measured $N\left(\mathrm{H}_{\mathrm{I}}\right)$ contour $[\log N(\mathrm{H} \mathrm{I})=15.8$; heavy triple-dot-dashed line $]$ envelopes most of the continuum shape-log $Z$ parameter space. However, we know that only an uncertain fraction of the measured $N(\mathrm{H}$ I) can coexist with the high-ionization gas. If at least half the measured $N(\mathrm{H} \mathrm{I})$ is in the lower ionization gas and $\alpha_{o x} \gtrsim-1.65$, then the lower limit to the RSF metallicity becomes $\mathrm{Z} \gtrsim 2.5 \mathrm{Z}_{\odot}$. This value increases to $4 \mathrm{Z}_{\odot}$ when only $10 \%$ of the measured neutral hydrogen coexists with the high-ionization gas. Note, too, that harder values of $\alpha_{o x}$ imply larger metallicities.

The predicted values of $N(\mathrm{C}$ III $), N(\mathrm{~N}$ III), and $N(\mathrm{O}$ III) are shown in Figures $3 e, 3 f$, and $3 g$, respectively. Like $N(\mathrm{H} \mathrm{I})$, these were not forced to match their measured column densities during the calculations. The continuum shape parameters are not constrained by the predicted doubly ionized column densities because those column densities are all less than their measured values (allowing for their $1 \sigma$ uncertainties; Table 1).

The likelihood that some of the C IV BAL forms with the lower ionization lines (and not with $\mathrm{N} \mathrm{V}$ or $\mathrm{O}$ vi) also pushes the solutions toward higher values of metallicity. Smaller $N(\mathrm{C}$ Iv $)$, for constant $N(\mathrm{~N}$ v) and $N(\mathrm{O} \mathrm{vI})$, moves the contours of constant metallicity toward the lower right in Figure $3 a$, increasing the area in continuum shape parameter space which requires high metallicities (the $\mathrm{H}$ I column density contours move toward the upper right in Figure $3 d$, and column density contours of the doubly ionized metals move toward the upper left in Figures $3 e, 3 f$, and $3 g)$. For example, if the $N(\mathrm{C}$ IV) in the high-ionization gas is only $30 \%$ of the measured value, then the contours shown in Figure $3 a$ and the upper and lower limits on the RSF metallicity take on values that are approximately 0.2 larger in $\log Z$. Thus, the inferred values of the RSF metallicities shown in Figure $3 a$ are conservative.

Figures $4 a-4 d$ show the results analogous to those in Figures $3 a-3 d$ for the $v^{-0.5}$ UV bump. Optimized fits to the measured $N(\mathrm{C} \mathrm{Iv}), N(\mathrm{~N} \mathrm{v})$, and $N(\mathrm{O} \mathrm{vI})$ yield values of $\log Z$, $\log U_{\text {bump }}$, and $\log N(\mathrm{H})$ for each pair of $\alpha_{o x}$ and $\log T_{\text {cutoff }}$ continuum shape parameters (Figs. 4a-4c). Most solutions involving $\alpha_{o x} \gtrsim-1.3$ and $\log T_{\text {cutoff }} \gtrsim 5.5$ produced significantly more $\mathrm{H}$ I than is measured in $0226-1024$. In addition, the quality of the fits to the $N(\mathrm{C}, \mathrm{N}, \mathrm{O})$ began to deteriorate just beyond these boundaries, especially in the direction of higher $\log T_{\text {cutoff }}$. As before, the fits broke down in the region of parameter space bounded by measured values of $\log N(\mathrm{H} \mathrm{I})$ and $1 \sigma$ above (between 15.8 and 15.9).

The results in Figure 4 are analogous to those in Figure 3, but the dependence on the continuum shape parameters differs because of the different character of the high-energy rolloff of the UV bump (see § 2.1.1). If a substantial fraction of the measured $N(\mathrm{H} \mathrm{I})$ resides in the lower ionization gas, the allowed values of the parameters would be restricted to regions with $\log T_{\text {cutoff }}<5.3$, or regions that are softer than the observed UV bump. Also, a smaller $N(\mathrm{C}$ IV) present in this gas would move the $N(\mathrm{H}$ I) contours to the right in Figure $4 d$ and the RSF metallicity contours downward in Figure $4 a$ (i.e., to larger values of $Z$ at a given position in the continuum-shape plane). The predicted doubly ionized metal column densities (not shown) do not strongly constrain the regions in the continuum-shape plane. Nonetheless, as was the case for the $v^{0.5} \mathrm{UV}$ bump, the metallicity must be at least solar.

Now we address the question of the sensitivity of the 

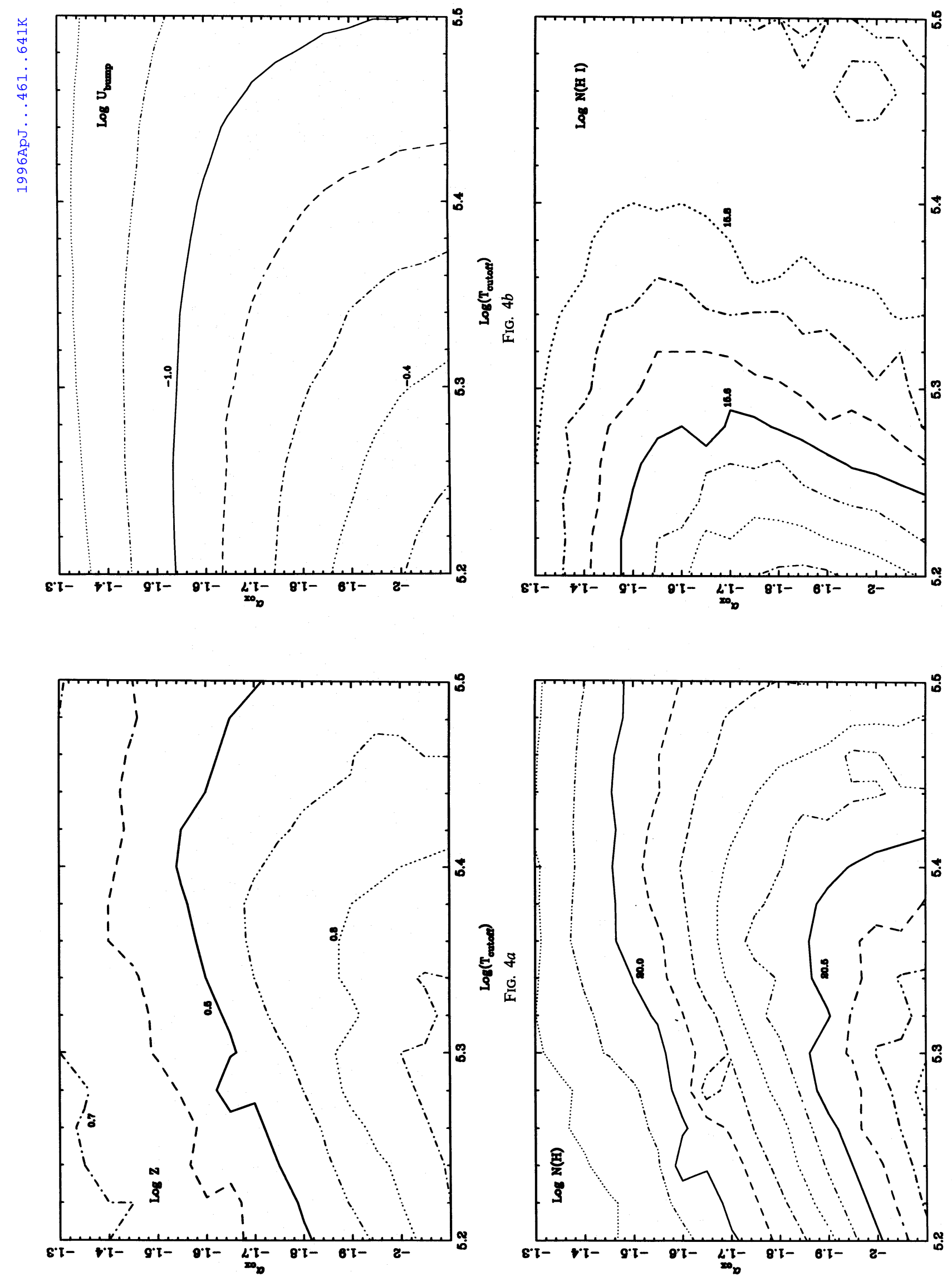

$:$

oi

$\Rightarrow$ 뭉

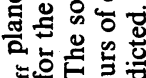

施

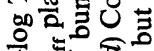

酸

on

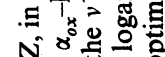

응

(

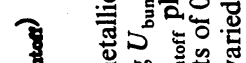

s.

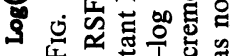

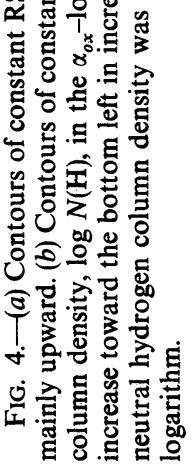


inferred RSF metallicities in Figures $3 a$ and $4 a$ to the unknown fraction of the observed $N(\mathrm{C}$ IV) and $N(\mathrm{H}$ I $)$ present in the high-ionization gas. We find that if the ratio of $N(\mathrm{H} \mathrm{I})$ actually present in the high-ionization gas to that calculated on Figures $3 d$ and $4 d$ is $x$ and if the ratio of the $N(\mathrm{C}$ IV) actually present in the high-ionization gas to the total measured value is $z$, then the metallicity contours in Figures $3 a$ and $4 a$ may be adjusted to $Z_{\mathrm{adj}} \approx z Z_{\mathrm{RSF}} / x$. The correction $x$ may arise (1) via the adjustment $z$, as described above, and/or (2) if the metallicities associated with the CNO abundance ratios differ from those given by the RSF enrichment scenario (see Table 2). The parameter $x$ is proportional to $z$ and inversely proportional to any adjustment in the metallicity scale in Table 2 . We will discuss the implications of point $(2)$ in $\$ 4$.

We conclude that the high-ionization column densities imply greater than solar metallicities, independent of the wide range of possible ionizing continuum shapes. The precise value of $Z$ one derives depends on the choice of continuum shape. The lower limit of $Z>Z_{\odot}$ is largely a consequence of the rapid decline in the relative nitrogen abundance at subsolar metallicities in the RSF enrichment scenario; lower metallicities would greatly underpredict the $N(\mathrm{~N} v)$. However, it is worth repeating that, even without this selective scaling of nitrogen, the result for $Z>Z_{\odot}$ also follows from comparing the overall metallic column densities to the upper limit on $\mathrm{H}_{\mathrm{I}}(\S 3.2)$.

\subsubsection{Predictions of $Z$ Based upon Doubly Ionized Metals}

The calculations above do not predict sufficient column densities in the doubly ionized metals. Therefore, we consider that these BALs form in gas with a lower ionization parameter, perhaps in gas with a higher particle density that is nearly cospatial with the high-ionization BAL gas.

When fitting the measured doubly ionized column densities, we used the measured $\mathrm{C}$ IV and $\mathrm{H}$ I column densities as upper limits and imposed an additional upper limit of $10^{14} \mathrm{~cm}^{-2}$ on the predicted $N(\mathrm{Mg}$ II) (unobserved). Because the ionization potentials of $\mathrm{C}$ III, $\mathrm{N}$ III, and $\mathrm{O}$ III are similar, the predicted metallicities are nearly independent of the ionizing continuum shape. Therefore, the column density of these lower ionization species should be the better indicator of $Z$, while those of higher ionization should constrain the continuum shape for the inferred $Z$. The predicted metallicities ranged from 0.8 to 1.0 , with most values lying above 0.9 , in $\log Z$. The predicted values of the ionization parameter of this gas were generally $\sim 1-1.5$ orders of magnitude smaller than those in the high-ionization gas, indicating a similar contrast in gas density if the two forms of gas are cospatial. We emphasize that our distinction between highly ionized and doubly ionized gas components is likely too simplistic.

There are two points to be made regarding these results. (1) The column densities of the doubly ionized metals have larger uncertainties, internal and possibly systematic. (2) As in the case of the high-ionization gas, we do not know how much of the measured $\mathrm{C}$ IV and $\mathrm{H}$ I column density belongs to which gas. These calculations generally predicted large fractions of the measured $N(\mathrm{C}$ IV $)$ but small fractions of the measured $N(\mathrm{~N} \mathrm{v})$ and $N(\mathrm{O} \mathrm{VI})$. This is consistent with our statement in the previous section that if a large fraction of the measured $N(\mathrm{C}$ IV) were not present in the highionization gas, larger metallicities and smaller $N\left(\begin{array}{ll}\mathrm{H} & \mathrm{I})\end{array}\right.$ would be predicted there (the RSF metallicity contours move generally downward in Figs. $3 a$ and $4 a$ ). The metallicities predicted in the two types of gas would then be more closely reconciled, with substantial enhancements over solar.

\subsubsection{All C, N, O Ions: The Single-Cloud Calculations}

Finally, we attempted to fit the full set of column densities in Table 1 from a single cloud. A perusal of Figures 3 and 4 indicates that such solutions, if they exist, would require very soft UV bumps and generally large values of $\alpha_{o x}$. In fact, we found that solutions based upon fitting all seven column densities do not fill up the continuum-shape plane. Rather, a very small region in ionizing continuum shape is permitted. Table 3 shows the results of such a fit for both UV bump types. All attempts at fitting the seven column densities led to parameters near these values. A large value of metallicity, consistent with the results of the highly and doubly ionized metal grids taken separately, is predicted. Although these parameters produced good fits to the seven BAL column densities of $0226-1024$, we do not consider the result likely, given the large predicted value of $\alpha_{o x}$ for the $v^{0.5} \mathrm{UV}$ bump and the extreme softness of the $v^{-0.5} \mathrm{UV}$ bump. We believe that this is indicative of the failure of one or more of the following: (1) the single-cloud model to describe correctly the ionization structure, (2) our description of the ionizing photon energy distribution, and (3) possibly our choice of chemical enrichment scenario in approximating the range of metallicities which could be present. We will discuss this last point in $\S 4$. In future work we will investigate the effects of shielding within the BAL flow due to hundreds of metal resonance lines which lie in the ionizing continuum (see second paragraph of Appendix C). Based upon the results of the present calculations, we speculate that larger values of $T_{\text {cutoff }}$ would be allowed if this shielding were included, and predicted values of $\alpha_{o x}$ could then be substantially smaller. Thus, the predicted continuum shape would be closer to that typically observed in QSOs. It is not entirely clear how this would effect the calculations of metallicity, since the amplitude of the shielding is dependent upon the metallicity. We speculate that the calculated RSF metallicity would be no larger than the present predictions (i.e., $Z \lesssim 10$ ), but this must be investigated in detail.

\section{SUMMARY AND DISCUSSION}

We have presented a detailed investigation of the ionization equilibrium and metallicity in BAL QSOs, based upon column densities of seven ions measured in 0226-1024. Given the large number of ill-constrained physical param-

TABLE 3

RESUlTS FOR Single-Cloud MODELS

\begin{tabular}{crr}
\hline \hline \multicolumn{1}{c}{ Parameter } & \multicolumn{1}{c}{$v^{0.5}$} & \multicolumn{1}{c}{$v^{-0.5}$} \\
\hline $\log T_{\text {cutoff }} \ldots \ldots \ldots$ & 4.96 & 4.80 \\
$\log U_{\text {bump }} \ldots \ldots \ldots$ & -1.66 & -1.57 \\
$\alpha_{\text {ox }} \ldots \ldots \ldots \ldots \ldots$ & -1.18 & -1.55 \\
$\log Z \ldots \ldots \ldots \ldots$ & 0.78 & 0.67 \\
$\log N(\mathrm{H}) \ldots \ldots \ldots$ & 19.66 & 19.84 \\
$\log N(\mathrm{H} \mathrm{I}) \ldots \ldots$ & 15.80 & 15.80 \\
$\log N(\mathrm{C}$ III $) \ldots \ldots$ & 15.76 & 15.82 \\
$\log N(\mathrm{C} \mathrm{IV}) \ldots \ldots$ & 16.20 & 16.22 \\
$\log N(\mathrm{~N}$ III $) \ldots \ldots$ & 16.15 & 16.09 \\
$\log N(\mathrm{~N}$ v) $\ldots \ldots$ & 16.34 & 16.26 \\
$\log N(\mathrm{O}$ III $) \ldots \ldots$ & 16.63 & 16.69 \\
$\log N(\mathrm{O}$ vI $) \ldots \ldots$ & 16.47 & 16.56 \\
\hline
\end{tabular}


eters, we have taken the approach of optimizing the fits to the measured column densities by varying three physical parameters $\left[U_{\text {bump }}, N(\mathrm{H})\right.$, and $\left.Z\right]$ for each of a wide range of ionizing continuum shapes. We considered the highly ionized and doubly ionized metals separately, as well as all ions taken together. We also consider two different metal enrichment scenarios: (1) simple scaled solar abundance ratios and (2) gas enriched via early-epoch rapid star formation in which nitrogen is selectively enhanced relative to carbon and oxygen. In all cases, the results indicate that metallicities in the BAL gas are greater than solar and could be as high as $5-10 \mathrm{Z}_{\odot}$. HF93 showed that the RSF evolution needed to reach these metallicities is consistent with expectations for the cores of massive galaxies.

The major difference between the RSF metallicity results and those using scaled solar calculations is that each metallicity has a unique contour in the continuum-shape plane in the former, while they overlap with one another in the latter. The reason for this difference is that while carbon and oxygen scale approximately with the metallicity in this chemical enrichment model (HF93), nitrogen scales as approximately its square. In other words, the selective enrichment of nitrogen predicted by the RSF enrichment can provide another very potent constraint on the overall metallicity of the gas. Furthermore, scaled solar abundances are probably not realistic representations of galactic chemical evolution. Therefore, the RSF-based calculations are both physically more plausible and more useful as diagnostics of the overall metal content.

A concern regarding the adoption of a particular enrichment scenario in this analysis is the following. Since the inferred metallicities in Figures $3 a$ and $4 a$ depend on the CNO abundance ratios (Table 2), how would the results change for different CNO ratios? Is there some combination of $\mathrm{CNO}$ ratios and continuum shapes that could yield much lower metallicities? Since the overall metallicities in RSF-based calculations (Figs. $3 a$ and $4 a$ ) are constrained by the relative nitrogen abundance, it is clear that larger $\mathrm{N} / \mathrm{C}$ and $\mathrm{N} / \mathrm{O}$ ratios at a given $Z$ would yield lower $Z$ results. However, there are several reasons to believe that the metallicities cannot be significantly lower than we already derived. First, ad hoc variations in $\mathrm{CNO}$ are not expected given the enrichment calculations of HF93 and others. In the particular models of HF93, the nitrogen yields were assumed to be near their maximum; so, if anything, we would expect lower relative nitrogen abundances resulting in still higher estimates of $Z$. Second, even for solar CNO abundance ratios, we showed that subsolar metallicities were not likely, given the constraints from the $N(\mathrm{H}$ I) and continuum shape. In addition, the large predicted $N(\mathrm{H} \mathrm{I})$ in Figures $3 d$ and (especially) $4 d$ and the predicted extreme softness of the UV bumps predicted in the single-cloud calculations both point to the possibility that the $\mathrm{Z} / \mathrm{H}$ abundance ratio in the RSF enrichment scenario is too small. That is, if anything, we are underestimating the metallicity as inferred from the present RSF abundances. Tests show that if we preserve the RSF metal abundance ratios, but then rescale the metal abundances by a single constant, solutions may be found which are analogous to those presented in Figures $3 a$ and $4 a$ (rescaled in $Z$ ), and the calculated $N(\mathrm{H} \mathrm{I})$ and $N(\mathrm{H})$ values change inversely with the change in the metallicity from that prescribed in the present RSF scenario. The constraint of the measured versus calculated $N(\mathrm{H} \mathrm{I})$ in the continuum shape plane (Figs. $3 d$ and $4 d$ ) leaves little possibility for $\mathrm{Z} / \mathrm{H}$ abundance ratios smaller than that adopted in this RSF enrichment scenario (model M5a of HF93). That the $\mathrm{Z} / \mathrm{H}$ ratio could be larger might indicate, for example, that the nitrogen yield might be smaller than that assumed in the present RSF enrichment scenario (\$ 3.3). Thus, we argue that our basic conclusion, that the BAL gas is metal enriched over solar, is not dependent upon the choice of this particular enrichment process.

Recently, HF92, HF93, and Ferland et al. (1996) presented evidence from the broad emission lines that the gas metallicities are above solar in QSOs. It is, therefore, not surprising that this would also be the case in the broad absorption-line region. We point out also that our results for above solar metallicities are consistent with studies of BALs by Turnshek (1988) and, most recently, Turnshek et al. (1996) using an entirely different (element-by-element) analysis. Using nearly the same data for 0226-1024, but only a small set of ionizing continuum shapes, Turnshek et al. found $(\mathrm{N} / \mathrm{C})$ and $(\mathrm{N} / \mathrm{O})$ ratios nearly identical to those in the $\mathrm{RSF}$ enrichment scenario for $\mathrm{Z}=10 \mathrm{Z}_{\odot}$, although they derive larger metal-to-hydrogen ratios.

It is worth noting that determining the metallicity from BALs has the advantage over the determination from broad emission lines in that the BALs yield directly the column densities of the various ions, prior to any ionization modeling. Modeling the emission-line gas, and thus determining its chemical abundances, requires some knowledge of the details of the macrophysics of the line-emitting region, such as geometry and line radiative transfer. It is, therefore, interesting that the emission- and absorption-line analyses are in good agreement. The case for high metallicities is thus clearly strengthened. As further evidence, the recent studies of QSO-associated absorption lines, at least some of which form in the near-QSO environment, also indicate $\mathrm{Z}>\mathrm{Z}_{\odot}$ (Møller, Jakobsen, \& Perryman 1994; Petitjean, Rauch, \& Carswell 1994; Hamann et al. 1995).

In addition to pointing to the host galaxy's chemical evolution, the knowledge of the metallicity of the BAL gas is important for another reason. Most dynamical calculations of BAL outflows have assumed solar metallicity. Metallicities enhanced over solar will increase the opacity of the outflowing BAL gas without significantly increasing the mass load of whatever is driving the outflow. This should have a significant impact on radiative acceleration models of BAL outflows (see Arav, Li, \& Begalman 1994; Baldwin et al. 1996).

Finally, we note that in spite of the growing evidence for high global metallicities, our understanding of the chemical abundances and the enrichment processes in BAL QSOs is far from complete. For example, if the identification of $\mathrm{P} v$, reported in two or three BAL QSOs thus far, proves correct, it will have profound implications on chemical enrichment scenarios and perhaps nucleosynthesis models. Currently, it is not understood how such a large overabundance in this element, $\mathrm{P} / \mathrm{C} \sim 60-80$ times solar (Junkkarinen et al. 1995), might arise. To our knowledge, such abundance anomalies are not predicted by any galaxy enrichment scenario. Furthermore, work remains to incorporate detailed models of the outflow, including the effects of multiple density cloudlets and BAL shielding of ionizing radiation, into calculations such as these. Observations of other BAL QSOs with many measurable column densities, as in the cases of 0226-1024 (K92) and 0946+3009 (Junkkarinen et al. 1995), need to be undertaken. Finally, better observational 
constraints on the shapes of ionizing continua in BAL QSOs should prove helpful to future analyses.

We gratefully acknowledge the helpful comments of Fred
Chaffee and an anonymous referee. K. K., J. F., and G. F. acknowledge support from NASA grant NAGW-3315 and NSF grant AST 93-19034. F. H. cknowledges support from NASA grant NAG5-1630 and AR5292.02-93A.

\section{APPENDIX A}

\section{OBSERVATIONAL CONSTRAINTS ON THE IONIZING CONTINUUM}

For simplicity we postulate that BAL QSOs belong to the parent population of radio-quiet QSOs (see W91), so that continuum shape properties are constrained by the observations of these well-studied objects. This may not be the case, however, since the absorbing gas may see a continuum whose spectral energy distribution differs from that observed in normal radio-quiet QSOs. Such differences could arise if the properties of the ionizing ultraviolet and X-ray continua are observer-orientation-dependent (see Netzer 1987) and if we observe the continua of BAL QSOs at preferential orientations relative to the geometry of the central accretion disk (e.g., along high inclination angles to the accretion disk). Unlike an analysis of broad emission lines in normal radio-quiet QSOs, where we are unsure whether the gas "sees" the observed continuum, the BAL gas should see the same continuum as the observer does (unless some part of the observed continuum forms at distances that are large compared to the BAL region). Unfortunately, the continuum properties of BAL QSOs are not well studied, and in any case the properties of the important extreme-ultraviolet (EUV) continuum are poorly constrained in all types of QSOs. The ionizing continuum in QSOs is generally characterized by two components: an ultraviolet "bump" (Malkan 1983) which rolls over to meet an X-ray power law. We will discuss briefly what is known about each in BAL QSOs.

W91 found that, on average, radio-quiet QSOs and BAL QSOs have very similar ultraviolet continuum shapes and emission-line properties (excluding those objects which exhibit low-ionization BALs). However, W91 did remark that below $\lambda_{\text {rest }} \sim 1200 \AA$, the BAL QSO continua seemed to soften relative to those of the normal radio-quiet sample. Indeed, K92 found that for the BAL QSO $0226-1024$, the continuum shape longward of $1300 \AA$, with a spectral index of about $-0.8\left(f_{v} \propto v^{\alpha}\right)$, nearly exactly matches that of the mean non-BAL QSO spectrum from W91, but becomes very steep, $\alpha \sim-5(!)$ shortward of $\sim 1200 \AA$. This slope differs substantially from the still relatively flat continuum observed down to approximately $900 \AA$ in the non-BAL QSO sample mean. The sudden plunge in the continuum may be caused by the Ly $\alpha$ forest (Møller \& Jakobsen 1990) or a soft UV bump, and K92 concluded that most of the steepness in the slope must be intrinsic to this BAL QSO. A subsequent shorter wavelength HST observation of this object indicates, however, that just shortward of $\sim 700 \AA$ the continuum flattens somewhat.

The X-ray data are available for only a few BAL QSOs and are generally of low signal-to-noise ratio (Bregman 1984; Gioia et al. 1986; Singh et al. 1987). However, PG 1416-129 is a bright, low-redshift BAL QSO for which the X-ray data are of high quality (ROSAT, de Kool, \& Meurs 1994; Ginga, Williams et al. 1992; Einstein, Tananbaum et al. 1986), and it is the only BAL QSO of which we are aware with a published $\alpha_{o x}(-1.38$; Boroson \& Green 1992). A $9700 \mathrm{~s}$ ROSAT observation of 0226- 1024 was attempted recently, but the 250 photons detected near the center of the field may have been from a nearby field object and not the intended target (Morris 1992). Recent ROSAT observations of other BAL QSOs indicate that BAL QSOs are, on average, observed to be more X-ray quiet than their normal QSO counterparts (Espey 1995; Green et al. 1995). In general, BAL QSOs are not expected to have significant intrinsic X-ray absorption, at least not from the BAL gas itself. De Kool \& Meurs (1994) derived a maximum column density of $\log N(\mathrm{H})=21.3$ in the BAL QSO PG 1416-129, assuming that the absorbing gas is of solar metallicity and is the same gas that produces the observed C IV and N v BALs. No Lyman limit systems have been observed in 0226-1024 down to a redshift of $\sim 0.8$. Thus, we do not expect that the soft X-rays are heavily absorbed, either within our Galaxy, within the object (whose rest-frame energies in the ROSAT band are $0.33-6.5 \mathrm{keV}$ ), or within the intergalactic medium. Observed values of $\alpha_{o x}$ for non-BAL QSOs range from -1 to about -2.1 , the average value is about -1.4 , and $\alpha_{o x}$ has been found to be correlated with the QSO luminosity such that more luminous QSOs tend to have steeper slopes (Zamorani et al. 1981; Avni \& Tananbaum 1986; Worrall et al. 1987; Mushotzky \& Wandel 1989; Bechtold et al. 1994; Wilkes et al. 1994). Using the relation given by the solid line in Figure 8 of Avni \& Tananbaum, we infer $\alpha_{o x} \approx-1.68$ for a QSO having the ultraviolet luminosity of $0226-1024$. The relation for radio-quiet QSOs in Worrall et al. gives the same result, while the relation in Wilkes et al. gives a value of -1.71 . However, we caution that there is a great deal of scatter, some of it probably intrinsic, about these relations, and it is possible that for BAL QSOs such a relation may not exist or is different. La Franca et al. (1995) dispute whether such a relation exists at all for QSOs. In summary, we consider the shape of the ionizing continuum in $0226-1024$, and in BAL QSOs in general, to be uncertain.

\section{APPENDIX B}

\section{ASSUMPTIONS REGARDING THE DERIVATION OF THE IONIC COLUMN DENSITIES AND THEIR ASSOCIATED SYSTEM UNCERTAINTIES}

Assuming that (1) the BAL gas completely covers the effective continuum source for all outflow velocities, (2) the BAL contains no unresolved, optically thick absorption lines, and (3) that scattered and/or collisionally excited emission from the BAL gas are negligible, then the optical depth $\tau$ is given by $\tau(\lambda)=-\ln \left[I_{r}(\lambda)\right]$, where $I_{r}(\lambda)$ is the residual intensity of the absorption line. We note that if any of these assumptions fail to apply, then the measured column densities become lower limits to their true values. Assumption (2) seems fairly secure in that BALs have been observed at echelle resolution and their 
residual intensities do not change. Assumption (3) may affect the BAL troughs at lower velocities. However, Hamann et al. (1993) investigated this question and found that low covering factors were required in most BAL QSOs, thus minimizing the possible effects of BAL emission filling in the troughs (see also Hamann \& Korista 1995). It is the first assumption which is the least certain. K92 showed evidence that incomplete coverage may play a role at some outflow velocities in $0226-1024$. Similar evidence has also been found in $1524+5147$ (Barlow 1995). If the coverage is not complete, it may be a function of outflow velocity, of the ion doing the absorbing, and of the energy of the ionizing or scattering photon if the effective continuum source is extended and its size or location differ with wavelength as seen by the BAL gas (Kwan 1990; K92). We define effective continuum to mean all photon emissions which may be scattered by the BAL gas, including those from broad emission lines. There is substantial evidence that the BAL gas covers a large fraction of the broad emission-line regions (see Turnshek et al. 1988). We note that the effects of differing covering fractions as a function of velocity are probably not important here because we consider column densities integrated over velocity. We also note that even if the BAL gas does not cover completely the effective continuum source, the results presented here will be changed little provided the covering fraction is independent of ionization and the actual larger column densities do not produce an ionization front through the gas. The former condition is almost certainly false; the highest ionizations BALs must cover more of the effective continuum than the lower ionization BALs (see K92). As for the latter condition, we find that the optical depth at the He II Lyman limit does not exceed unity until the $\mathrm{C}$ IV, $\mathrm{N} v$, and $\mathrm{O}$ vi column densities take on values greater than 3 times their measured values, for a given metallicity.

\section{APPENDIX C}

\section{THE ATTENUATION OF IONIZING RADIATION INSIDE AND OUTSIDE THE BAL FLOW}

When $\lambda_{\text {rest }}=912 \AA$ can be observed, it is found that the gas in non-Mg II BAL QSOs is optically thin at the hydrogen Lyman limit. However, in general, the ionization structure within the BAL region is poorly understood. The present application of CLOUDY to investigate this ionization structure is not altogether appropriate, since it assumes the clouds to be static, whereas huge velocity gradients must be present in the BAL region. The line emission from such a region would depend heavily on the geometry and velocity field of the emitting gas (see Hamann et al. 1993). However, the ionization equilibrium which governs the predicted column densities is less sensitive to the details of the velocity gradients. The shifting in velocity of the ionization edges important in shielding results in errors in the photoionization rate whose magnitude is $\sim v / c$. Larger uncertainties are present for $\mathrm{He}$ II near the $\mathrm{He}$ II recombination front and $\mathrm{H}$ i near the hydrogen recombination front, if these fronts are present. However, ionization, edges, either valence or $\mathrm{K}$ shell, should be weak in the high-ionization BAL gas discussed here, but are important to calculations of Mg II-type BAL QSOs (Voit et al. 1993). For example, all the calculations above predict optical depths at the He II Lyman limit of $\tau(4$ ryd $)<1$.

Another kind of shielding may be important to the ionization equilibrium within the BAL flow-that due to overlapping BALs expected from hundreds of resonance lines lying within the ionizing continuum $(\lambda<912 \AA)$. The cumulative absorption may be significant enough to affect the ionization of the flow downstream. The magnitude of this effect will be sensitive to the metallicity of the gas, since nearly all the opacity arises from metal BALs and will almost certainly be energy dependent. Given the stages of ionization observed to exist within the BAL flow, the strongest resonance lines appear mainly above $228 \AA$; however, the number of lines grows drastically below $228 \AA$. The effect of this attenuation has never been investigated in detail and is not taken into account in the calculations presented here; it is the subject of future work.

Another possible effect which could change the ionization equilibrium in the BAL gas is if there is substantial shielding at energies $\sim 0.1-2 \mathrm{keV}$ from gas lying between the ionizing continuum and the BAL region, as suggested in a model for the BAL flow by Murray et al. (1995). This absorption, if it exists, must be tuned such that there is very little opacity below $120 \mathrm{eV}$, or we would never observe an O VI BAL. In this paper, we assume that the surface of the BAL region nearest the continuum source sees an unattenuated continuum, as described in $\S 2.1 .1$.

Arav, N., Li, Z.-Y., \& Begelman, M. C. 1994, ApJ, 432, 62

Arimoto, N., \& Yoshii, Y. 1986, A\&A, 164, 260

Avni, Y., \& Tananbaum, H. 1986, ApJ, 305, 83

Baldwin, J. A., et al. 1996, ApJ, in press

Barlow, T. 1995, private communication

Barvainis, R. 1990, ApJ, 353, 419

Bechtold, J., et al. 1994, AJ, 108, 374

Bica, E. 1988, A\&A, 195, 76

Bica, E., Arimoto, N., \& Alloin, D. 1988, A\&A, 202, 8

Boroson, T. A., \& Green, R. F. 1992, ApJS, 80, 109

Bregman, J. N. 1984, ApJ, 276, 423

Chaffee, F. H., Foltz, C. B., Hewett, P. C., Francis, P. J., Weymann, R. J., Morris, S. L., Anderson, S. F., \& MacAlpine, G. M. 1991, AJ, 102, 461

de Kool, M., \& Meurs, E. J. A. 1994, A\&A, 281, L65

Espey, B. 1995, private communication

Ferland, G. J. 1994, HAZY, a Brief Introduction to Cloudy (Univ. Kentucky Phys. Dept. Internal Report)

Ferland, G. J., Baldwin, J. A., Korista, K. T., Hamann, F., Carswell, R. F., Phillips, M., Wilkes, B., \& Williams, R. E. 1996, ApJ, in press

Ferland, G. J., \& Persson, S. E. 1989, ApJ, 347, 656

Ferland, G. J., Peterson, B. M., Horne, K., Welsh, W. F., \& Nahar, S. N. 1992, ApJ, 387, 95

\section{REFERENCES}

Foltz, C. B., Chaffee, F. H., Hewett, P. C., MacAlpine, G. M., Turnshek, D. A., Weymann, R. J., \& Anderson, S. F. 1987, AJ, 94, 1423

Foltz, C. B., Chaffee, F. H., Hewett, P. C., Weymann, R. J., \& Anderson, S. F., \& MacAlpine, G. M. 1989, AJ, 98, 1959

Francis, P. J. 1993, ApJ, 407, 519

Geisler, D., Friel, E. D. 1992, AJ, 104, 128

Gioia, I. M., Maccacaro, T., Schild, R. E., Giommi, P., \& Stocker, J. T. 1986, ApJ, 307, 497

Green, P. J., et al. 1995, ApJ, 450, 51

Grevesse, N., \& Anders, E. 1989, in AIP Conf. Proc. 183, Cosmic Abundances of Matter, ed. C. J. Waddington (New York: AIP), 1

Hamann, F., Barlow, T., Beaver, E., Burbidge, E. M., Cohen, R., Junkkarinen, V., \& Lyons, R. 1995, ApJ, 443, 606

Hamann, F., \& Ferland, G. J. 1992, ApJ, 391, L53 (HF92)

. 1993, ApJ, 418, 11 (HF93)

Hamann, F., \& Korista, K. T. 1995, ApJ, submitted

Hamann, F., Korista, K. T., \& Morris, S. L. 1993, ApJ, 415, 541

Junkkarinen, V. T., Beaver, E. A., Burbidge, E. M., Cohen, R. W., Hamann, F., Lyons, R. W., \& Barlow, T. A. 1995, BAAS, 27, 872

Korista, K. T., Voit, G. M., Morris, S. L., \& Weymann, R. J. 1993, ApJS, 88,357

Korista, K. T., et al. 1992, ApJ, 401, 529 (K92) 
Smith, D. A., Done, C., \& Pounds, K. A. 1993, MNRAS, 263, 54

Stocke, J., Morris, S., Weymann, R., \& Foltz, C. 1992, ApJ, 396, 487

Sun, W.-H., \& Malkan, M. A. 1989, ApJ, 346, 68

Tananbaum, H., Avni, Y., Green, R., Schmidt, M., \& Zamorani, G. 1986, ApJ, 305, 57

Turnshek, D. A. 1984, ApJ, 278, L87

. 1988, in QSO Absorption Lines: Probing the Universe, ed. J. C. Blades, C. Norman, \& D. A. Turnshek (Cambridge: Cambridge Univ. Press), 17

Turnshek, D. A., Briggs, F. H., Foltz, C. B., Grillmair, C. J., \& Weymann, R. J. 1987, unpublished paper

Turnshek, D. A., Foltz, C. B., Grillmair, C. J., \& Weymann, R. J. 1988, ApJ, 325,651

Turnshek, D. A., Kopko, M., Jr., Monier, E., Noll, D., Espey, B., \& Weymann, R. J. 1996, ApJ, submitted

Voit, G. M., Weymann, R. J., \& Korista, K. T. 1993, ApJ, 413, 95

Wampler, E. J., Chugai, N. N., \& Pettitjean, P. 1995, ApJ, 443, 586

Weymann, R. J., Morris, S. L., Foltz, C. B., \& Hewett, P. C. 1991, ApJ, 373, 23 (W91)

Weymann, R. J., Turnshek, D. A., \& Christiansen, W. A. 1985, in Astrophysics of Active Galaxies and Quasi-Stellar Objects, ed. J. S. Miller (Mill Valley: University Science Books), 333 (WTC85)

Wilkes, B. J., Tananbaum, H., Worrall, D. M., \& Avni, Y. 1994, ApJS, 92, 53

Williams, O. R., et al. 1992, ApJ, 389, 157

Worrall, D. M., Giommi, P., Tananbaum, H., \& Zamorani, G. 1987, ApJ, 313,596

Zamorani, G., et al. 1981, ApJ, 245, 357 